\title{
Limit-point buckling analyses using solid, shell and solid-shell elements
}

\author{
Marc Killpack $^{\text {a,b }}$, Farid Abed-Meraim ${ }^{\text {a,* }}$ \\ ${ }^{a}$ LEM3, UMR CNRS 7239, Arts et Métiers ParisTech, \\ 4 rue A. Fresnel, 57078 Metz Cedex 03, France \\ ${ }^{\mathrm{b}}$ Georgia Tech Lorraine, Georgia Institute of Technology, \\ 2-3 rue Marconi, 57070 Metz, France
}

\begin{abstract}
In this paper, the recently-developed solid-shell element SHB8PS is used for the analysis of a representative set of popular limit-point buckling benchmark problems. For this purpose, the element has been implemented in Abaqus/Standard finite element software and the modified Riks method was employed as an efficient path-following strategy. For the benchmark problems tested, the new element shows better performance compared to solid elements and often performs as well as state-of-the-art shell elements. In contrast to shell elements, it allows for the accurate prescription of boundary conditions as applied to the actual edges of the structure.
\end{abstract}

Keywords: Assumed-strain; Solid-shell; Locking; Limit-point buckling; Boundary conditions; Benchmark problems

\footnotetext{
* Corresponding author: farid.abed-meraim@ensam.eu (F. Abed-Meraim), Tel: +(33) 3.87.37.54.79, Fax: $+(33)$ 3.87.37.54.70.
} 


\section{Introduction}

Traditionally, shell finite elements are best suited for the numerical simulation of thin structure applications, while solid elements are regularly used for bulk structures. However, engineering structures often combine thin components with thick/bulk geometries in the same assembly. Thus, the finite element modeling of such applications would be considerably simplified if the same type of finite element could be successfully used in both zones. Alternatively, solid and shell elements can be mixed in the same model, but special effort is required for matching the translations in solid elements and rotations in shell elements. This laborious task generally involves defining algebraic constraints at the interface or introducing solid-to-shell transition elements which often result in excessively stiff behavior. In addition, most shell formulations rely on specific kinematic descriptions along with particular constitutive assumptions, which require the additional effort of implementation of plane-stress behavior models.

In order to overcome the aforementioned limitations, solid-shell elements have recently emerged as an interesting alternative. Indeed, these elements combine a shell-like response with three-dimensional element geometry, thus naturally matching solid elements in the same mesh. Numerous developments have been made in this direction during the last decade (see, e.g., Domissy [1], Cho et al. [2], Hauptmann and Schweizerhof [3], Lemosse [4], Sze and Yao [5], Hauptmann et al. [6]). The SHB8PS element is one such recently developed element that is based on a purely three-dimensional approach (Abed-Meraim and Combescure [7,8], Legay and Combescure [9]). Note that most of the methods developed earlier were based on the enhanced assumed strain method proposed by Simo and coworkers (Simo and Rifai [10], Simo and Armero [11], Simo et al. [12]), and consisted of either the use of a conventional integration scheme with appropriate control of all locking phenomena or the application of a reduced integration technique with associated hourglass control. Both approaches have been extensively investigated and evaluated in various structural applications, as reported in recent contributions by Vu-Quoc and Tan [13], Chen and Wu [14], Kim et al. [15], Alves de Sousa et al. [16,17], Reese [18], and Abed-Meraim and Combescure [19].

In spite of its three-dimensional geometry (eight-node hexahedron), the SHB8PS element has received specific treatments and enhancements so that it exhibits the desirable shell features in structural applications. The standard three-dimensional constitutive law is modified such that plane-stress assumptions are approached and the integration points are aligned along a preferential direction designated as the "thickness". Reduced integration is employed in order to improve the element's computational efficiency and to alleviate membrane and shear locking. The hourglass modes that are thus inherently induced are physically stabilized following the efficient approach of Belytschko and Bindeman [20]. In order to eliminate the various locking phenomena (transverse shear, membrane, thickness), 
the discrete gradient operator is projected onto an appropriate sub-space. This projection technique can be expressed under the formalism of the assumed strain method and justified within the framework of the $\mathrm{Hu}$-Washizu three-field variational principle. The recent projection of the discrete gradient operator adopted in Abed-Meraim and Combescure [19] shows locking-free response and optimal convergence in various linear as well as nonlinear benchmark problems.

More specifically, the motivation behind the development of the SHB8PS solid-shell element is to combine in a single formulation the advantages of both solid and shell elements. Some of the resulting benefits are summarized below:

- Ability to model thin, three-dimensional structures using a single layer of elements along the thickness, while accurately describing the various through-thickness phenomena.

- Simplified meshing of complex geometries, in which structural and continuum elements must coexist, without any compatibility problems between different families of elements.

- Easy treatment of large rotations by a straightforward configuration update (with no rotational degrees of freedom involved) when compared to shell element formulations.

- Computational efficiency due to large admissible aspect ratios, the use of reduced integration, and elimination of shear and membrane locking by appropriate techniques.

- Simple and attractive formulation (hexahedral geometry, eight nodes, only three translational degrees of freedom per node), thus avoiding complex shell formulations.

The aim of this paper is to investigate the ability of the recently developed solid-shell element SHB8PS to predict limit-point buckling phenomena for thin structures. Because buckling is known to be a strongly nonlinear phenomenon involving large rotations and deflections, a path-following strategy is needed for tracking the solutions beyond the limitpoint. For this purpose, the SHB8PS element has been implemented in the finite element software Abaqus/Standard and the modified Riks method has been used.

The remainder of the paper is structured as follows. The formulation of the SHB8PS solid-shell element is briefly described in Section 2, together with some general comments on limit-point buckling and the modified Riks method. In Section 3, a representative set comprising six limit-point buckling benchmark tests is thoroughly investigated to compare the respective performance of the SHB8PS element and Abaqus linear solid, shell and solid-shell elements. In Section 4, these solutions are shown to be sensitive to the particular location of boundary conditions along the thickness (on the upper edge, lower edge or mid-line, respectively). It is shown, in particular, that elements with threedimensional geometry - including SHB8PS - can be advantageously used to model all of these situations. 


\section{Formulation of the SHB8PS finite element and Riks' modified method}

\subsection{Formulation of the assumed strain physically stabilized solid-shell element SHB8PS}

This section gives a short description of the SHB8PS element formulation. Only an outline is provided since the detailed derivation can be found in References $[8,19]$.

\subsubsection{Finite element interpolation and discrete gradient operator}

SHB8PS is a hexahedral, eight-node, isoparametric element with linear interpolation. It has three displacement degrees of freedom per node and a set of $n_{\text {int }}$ integration points spread along the $\zeta$ direction in the local coordinate frame (see Fig. 1). The coordinates $x_{i}$, $i=1,2,3$ of a point in the element are related to the nodal coordinates $x_{i I}$ using the classical tri-linear shape functions $\mathbf{N}^{T}=\left(N_{1}, N_{2}, \ldots, N_{8}\right)$ :

$$
x_{i}=x_{i I} N_{I}(\xi, \eta, \zeta)=\sum_{I=1}^{8} x_{i I} N_{I}(\xi, \eta, \zeta)
$$

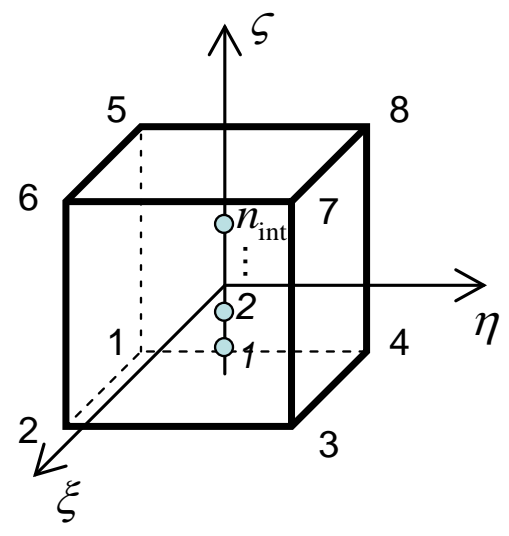

\begin{tabular}{cccc}
\hline node & $\xi$ & $\eta$ & $\zeta$ \\
\hline 1 & -1 & -1 & -1 \\
2 & 1 & -1 & -1 \\
3 & 1 & 1 & -1 \\
4 & -1 & 1 & -1 \\
5 & -1 & -1 & 1 \\
6 & 1 & -1 & 1 \\
7 & 1 & 1 & 1 \\
8 & -1 & 1 & 1 \\
\hline
\end{tabular}

Fig. 1. SHB8PS reference geometry, integration point locations and nodal coordinates.

The displacement field interpolation, which is similar to Eq. (1), together with the expression of the tri-linear shape functions can be rearranged in the following form:

$$
u_{i}=\left(a_{0 i}+x_{1} \mathbf{b}_{1}^{T}+x_{2} \mathbf{b}_{2}^{T}+x_{3} \mathbf{b}_{3}^{T}+h_{1} \gamma_{1}^{T}+h_{2} \boldsymbol{\gamma}_{2}^{T}+h_{3} \boldsymbol{\gamma}_{3}^{T}+h_{4} \boldsymbol{\gamma}_{4}^{T}\right) \cdot \mathbf{d}_{i}
$$

where: 


$$
\left\{\begin{array}{l}
\mathbf{b}_{i}=\mathbf{N}_{, i}(\mathbf{0})=\frac{\partial \mathbf{N}}{\partial x_{i} \mid \xi=\eta=\zeta=0} \quad, \quad i=1,2,3 \\
h_{1}=\eta \zeta, h_{2}=\zeta \xi, h_{3}=\xi \eta, h_{4}=\xi \eta \zeta \\
\boldsymbol{\gamma}_{\alpha}=\frac{1}{8}\left[\mathbf{h}_{\alpha}-\sum_{j=1}^{3}\left(\mathbf{h}_{\alpha}^{T} \cdot \mathbf{x}_{j}\right) \mathbf{b}_{j}\right] \quad, \alpha=1, \ldots, 4
\end{array}\right.
$$

In the above equations, the $\mathbf{d}_{i}$ and $\mathbf{x}_{i}$ vectors indicate the nodal displacements and coordinates and are defined as:

$$
\left\{\begin{array}{l}
\mathbf{d}_{i}^{T}=\left(u_{i 1}, u_{i 2}, u_{i 3}, \ldots ., u_{i 8}\right) \\
\mathbf{x}_{i}^{T}=\left(x_{i 1}, x_{i 2}, x_{i 3}, \ldots ., x_{i 8}\right)
\end{array}\right.
$$

while vectors $\mathbf{h}_{\alpha}(\alpha=1, \ldots, 4)$ are given by:

$$
\left\{\begin{array}{l}
\mathbf{h}_{1}^{T}=(1,1,-1,-1,-1,-1,1,1) \\
\mathbf{h}_{2}^{T}=(1,-1,-1,1,-1,1,1,-1) \\
\mathbf{h}_{3}^{T}=(1,-1,1,-1,1,-1,1,-1) \\
\mathbf{h}_{4}^{T}=(-1,1,-1,1,1,-1,1,-1)
\end{array}\right.
$$

This allows us to express the discrete gradient operator relating the strain field to the nodal displacements. With the convention of implied summation for repeated subscripts it reads:

$$
\begin{aligned}
& \nabla_{s}(\mathbf{u})=\mathbf{B} \cdot \mathbf{d} \\
& \mathbf{B}=\left[\begin{array}{ccc}
\mathbf{b}_{x}^{T}+h_{\alpha, x} \boldsymbol{\gamma}_{\alpha}^{T} & \mathbf{0} & \mathbf{0} \\
\mathbf{0} & \mathbf{b}_{y}^{T}+h_{\alpha, y} \boldsymbol{\gamma}_{\alpha}^{T} & \mathbf{0} \\
\mathbf{0} & \mathbf{0} & \mathbf{b}_{z}^{T}+h_{\alpha, z} \boldsymbol{\gamma}_{\alpha}^{T} \\
\mathbf{b}_{y}^{T}+h_{\alpha, y} \boldsymbol{\gamma}_{\alpha}^{T} & \mathbf{b}_{x}^{T}+h_{\alpha, x} \boldsymbol{\gamma}_{\alpha}^{T} & \mathbf{0} \\
\mathbf{0} & \mathbf{b}_{z}^{T}+h_{\alpha, z} \boldsymbol{\gamma}_{\alpha}^{T} & \mathbf{b}_{y}^{T}+h_{\alpha, y} \boldsymbol{\gamma}_{\alpha}^{T} \\
\mathbf{b}_{z}^{T}+h_{\alpha, z} \boldsymbol{\gamma}_{\alpha}^{T} & \mathbf{0} & \mathbf{b}_{x}^{T}+h_{\alpha, x} \boldsymbol{\gamma}_{\alpha}^{T}
\end{array}\right]
\end{aligned}
$$

\subsubsection{Hu-Washizu variational principle and assumed strain field}

The discrete gradient operator is projected onto an appropriate sub-space in order to eliminate shear and membrane locking. This projection technique follows the assumed strain method detailed by Belytschko and Bindeman [20]. This approach can also be justified within the framework of the $\mathrm{Hu}-\mathrm{Washizu}$ nonlinear mixed variational principle expressed as: 


$$
\pi(\mathbf{v}, \dot{\overline{\boldsymbol{\varepsilon}}}, \overline{\boldsymbol{\sigma}})=\int_{\Omega_{e}} \delta \dot{\overline{\boldsymbol{\varepsilon}}}^{T} \cdot \boldsymbol{\sigma} d \Omega+\delta \int_{\Omega_{e}} \overline{\boldsymbol{\sigma}}^{T} \cdot\left(\nabla_{s}(\mathbf{v})-\dot{\overline{\boldsymbol{\varepsilon}}}\right) d \Omega-\delta \dot{\mathbf{d}}^{T} \cdot \mathbf{f}^{e x t}=0
$$

where $\delta$ denotes a variation, $\mathbf{v}$ the velocity field, $\dot{\overline{\boldsymbol{\varepsilon}}}$ the assumed strain rate, $\overline{\boldsymbol{\sigma}}$ the interpolated stress, $\boldsymbol{\sigma}$ the stress evaluated by the constitutive law, and $\mathbf{f}^{\text {ext }}$ the external nodal forces. The assumed strain formulation used to develop the SHB8PS element is a simplified form of the $\mathrm{Hu}-$ Washizu variational principle as described by Simo and Hughes [21]. In this simplified form, the interpolated stress is chosen to be orthogonal to the difference between the symmetric part of the velocity gradient and the assumed strain rate. Consequently, the second term in the right-hand side of Eq. (7) vanishes, yielding:

$$
\pi(\dot{\overline{\boldsymbol{\varepsilon}}})=\int_{\Omega_{e}} \delta \dot{\overline{\boldsymbol{\varepsilon}}}^{T} \cdot \boldsymbol{\sigma} d \Omega-\delta \dot{\mathbf{d}}^{T} \cdot \mathbf{f}^{e x t}=0
$$

In this form, the variational principle is independent of the stress interpolation, since the interpolated stress is eliminated and no longer needs to be defined. The discrete equations then only require the interpolation of the velocity and of the assumed strain field. The assumed strain rate $\dot{\overline{\boldsymbol{\varepsilon}}}$ is expressed in terms of a $\overline{\mathbf{B}}$ matrix, projected starting from the classical discrete gradient $\mathbf{B}$ defined by Eq. (6):

$$
\dot{\bar{\varepsilon}}(x, t)=\overline{\mathbf{B}}(x) \cdot \dot{\mathbf{d}}(t)
$$

Once this expression is substituted into the variational principle (8), the expressions of the elastic stiffness and internal forces are obtained:

$$
\mathbf{K}_{e}=\int_{\Omega_{e}} \overline{\mathbf{B}}^{T} \cdot \mathbf{C} \cdot \overline{\mathbf{B}} d \Omega \quad, \quad \mathbf{f}^{i n t}=\int_{\Omega_{e}} \overline{\mathbf{B}}^{T} \cdot \boldsymbol{\sigma}(\dot{\overline{\boldsymbol{\varepsilon}}}) d \Omega
$$

Before defining the projected $\overline{\mathbf{B}}$ operator, in the previous equations, vectors $\mathbf{b}_{i}$ defined in Eq. (3) are replaced by the mean form $\hat{\mathbf{b}}_{i}$ proposed by Flanagan and Belytschko [22]:

$$
\hat{\mathbf{b}}_{i}=\frac{1}{\Omega_{e}} \int_{\Omega_{e}} \mathbf{N}_{, i}(\xi, \eta, \zeta) d \Omega, \quad i=1,2,3
$$

Accordingly, vectors $\gamma_{\alpha}$ are replaced by vectors $\hat{\gamma}_{\alpha}$ defined as:

$$
\hat{\boldsymbol{\gamma}}_{\alpha}=\frac{1}{8}\left[\mathbf{h}_{\alpha}-\sum_{j=1}^{3}\left(\mathbf{h}_{\alpha}^{T} \cdot \mathbf{x}_{j}\right) \hat{\mathbf{b}}_{j}\right]
$$

and matrix B , defined in Eq. (6), is replaced by the $\hat{\mathbf{B}}$ operator, defined as: 


$$
\hat{\mathbf{B}}=\left[\begin{array}{ccc}
\hat{\mathbf{b}}_{x}^{T}+h_{\alpha, x} \hat{\boldsymbol{\gamma}}_{\alpha}^{T} & \mathbf{0} & \mathbf{0} \\
\mathbf{0} & \hat{\mathbf{b}}_{y}^{T}+h_{\alpha, y} \hat{\boldsymbol{\gamma}}_{\alpha}^{T} & \mathbf{0} \\
\mathbf{0} & \mathbf{0} & \hat{\mathbf{b}}_{z}^{T}+h_{\alpha, z} \hat{\boldsymbol{\gamma}}_{\alpha}^{T} \\
\hat{\mathbf{b}}_{y}^{T}+h_{\alpha, y} \hat{\boldsymbol{\gamma}}_{\alpha}^{T} & \hat{\mathbf{b}}_{x}^{T}+h_{\alpha, x} \hat{\boldsymbol{\gamma}}_{\alpha}^{T} & \mathbf{0} \\
\mathbf{0} & \hat{\mathbf{b}}_{z}^{T}+h_{\alpha, z} \hat{\boldsymbol{\gamma}}_{\alpha}^{T} & \hat{\mathbf{b}}_{y}^{T}+h_{\alpha, y} \hat{\boldsymbol{\gamma}}_{\alpha}^{T} \\
\hat{\mathbf{b}}_{z}^{T}+h_{\alpha, z} \hat{\boldsymbol{\gamma}}_{\alpha}^{T} & \mathbf{0} & \hat{\mathbf{b}}_{x}^{T}+h_{\alpha, x} \hat{\boldsymbol{\gamma}}_{\alpha}^{T}
\end{array}\right]
$$

At this stage, the above operator $\hat{\mathbf{B}}$ is projected onto the following $\overline{\hat{\mathbf{B}}}$ matrix:

$$
\overline{\hat{\mathbf{B}}}=\left[\begin{array}{ccc}
\hat{\mathbf{b}}_{x}^{T}+\sum_{\alpha=1}^{4} h_{\alpha, x} \hat{\boldsymbol{\gamma}}_{\alpha}^{T} & \mathbf{0} & \mathbf{0} \\
\mathbf{0} & \hat{\mathbf{b}}_{y}^{T}+\sum_{\alpha=1}^{4} h_{\alpha, y} \hat{\boldsymbol{\gamma}}_{\alpha}^{T} & \mathbf{0} \\
\mathbf{0} & \mathbf{0} & \hat{\mathbf{b}}_{z}^{T}+\sum_{\alpha=1}^{3} h_{\alpha, z} \hat{\gamma}_{\alpha}^{T} \\
\hat{\mathbf{b}}_{y}^{T}+\sum_{\alpha=1}^{2} h_{\alpha, y} \hat{\boldsymbol{\gamma}}_{\alpha}^{T} & \hat{\mathbf{b}}_{x}^{T}+\sum_{\alpha=1}^{2} h_{\alpha, x} \hat{\boldsymbol{\gamma}}_{\alpha}^{T} & \mathbf{0} \\
\mathbf{0} & \hat{\mathbf{b}}_{z}^{T}+\sum_{\alpha=1,2}^{2} h_{\alpha, z} \hat{\boldsymbol{\gamma}}_{\alpha}^{T} & \hat{\mathbf{b}}_{y}^{T}+\sum_{\alpha=1}^{2} h_{\alpha, y} \hat{\gamma}_{\alpha}^{T} \\
\hat{\mathbf{b}}_{z}^{T}+\sum_{\alpha=1}^{2} h_{\alpha, z} \hat{\boldsymbol{\gamma}}_{\alpha}^{T} & \mathbf{0} & \hat{\mathbf{b}}_{x}^{T}+\sum_{\alpha=1,2,4}^{h_{\alpha, x}} \hat{\gamma}_{\alpha}^{T}
\end{array}\right]
$$

\subsubsection{Stabilization of spurious zero-energy modes}

For the SHB8PS element, the spurious modes are shown to originate in the particular location of the integration points (along a line). For a set of $n_{\text {int }}$ integration points $\left(J=1, \ldots, n_{\text {int }}\right)$, with coordinates $\xi_{J}=\eta_{J}=0, \zeta_{J} \neq 0$, the derivatives $h_{\alpha, i}(\alpha=3,4 ; i=1,2,3)$ vanish. Consequently, for $n_{\text {int }} \geq 2$ operator $\overline{\hat{\mathbf{B}}}$ defined by Eq. (14) reduces to matrix $\hat{\mathbf{B}}_{12}$, where the sum on the index $\alpha$ only ranges from 1 to 2 :

$$
\hat{\mathbf{B}}_{12}=\left[\begin{array}{ccc}
\hat{\mathbf{b}}_{x}^{T}+\sum_{\alpha=1}^{2} h_{\alpha, x} \hat{\boldsymbol{\gamma}}_{\alpha}^{T} & \mathbf{0} & \mathbf{0} \\
\mathbf{0} & \hat{\mathbf{b}}_{y}^{T}+\sum_{\alpha=1}^{2} h_{\alpha, y} \hat{\boldsymbol{\gamma}}_{\alpha}^{T} & \mathbf{0} \\
\mathbf{0} & \mathbf{0} & \hat{\mathbf{b}}_{z}^{T}+\sum_{\alpha=1}^{2} h_{\alpha, z} \hat{\boldsymbol{\gamma}}_{\alpha}^{T} \\
\hat{\mathbf{b}}_{y}^{T}+\sum_{\alpha=1}^{2} h_{\alpha, y} \hat{\boldsymbol{\gamma}}_{\alpha}^{T} & \hat{\mathbf{b}}_{x}^{T}+\sum_{\alpha=1}^{2} h_{\alpha, x} \hat{\boldsymbol{\gamma}}_{\alpha}^{T} & \mathbf{0} \\
\mathbf{0} & \hat{\mathbf{b}}_{z}^{T}+\sum_{\alpha=1,2}^{\alpha} h_{\alpha, z} \hat{\boldsymbol{\gamma}}_{\alpha}^{T} & \hat{\mathbf{b}}_{y}^{T}+\sum_{\alpha=1}^{2} h_{\alpha, y} \hat{\boldsymbol{\gamma}}_{\alpha}^{T} \\
\hat{\mathbf{b}}_{z}^{T}+\sum_{\alpha=1}^{2} h_{\alpha, z} \hat{\boldsymbol{\gamma}}_{\alpha}^{T} & \mathbf{0} & \hat{\mathbf{b}}_{x}^{T}+\sum_{\alpha=1,2}^{h_{\alpha, x} \hat{\boldsymbol{\gamma}}_{\alpha}^{T}}
\end{array}\right]
$$


A detailed analysis of stiffness matrix rank deficiency has been carried out in [8,19], which revealed the following six hourglass modes:

$$
\left(\begin{array}{c}
\mathbf{h}_{3} \\
\mathbf{0} \\
\mathbf{0}
\end{array}\right),\left(\begin{array}{c}
\mathbf{0} \\
\mathbf{h}_{3} \\
\mathbf{0}
\end{array}\right),\left(\begin{array}{c}
\mathbf{0} \\
\mathbf{0} \\
\mathbf{h}_{3}
\end{array}\right),\left(\begin{array}{c}
\mathbf{h}_{4} \\
\mathbf{0} \\
\mathbf{0}
\end{array}\right),\left(\begin{array}{c}
\mathbf{0} \\
\mathbf{h}_{4} \\
\mathbf{0}
\end{array}\right),\left(\begin{array}{c}
\mathbf{0} \\
\mathbf{0} \\
\mathbf{h}_{4}
\end{array}\right)
$$

The control of the above hourglass modes for the SHB8PS element is achieved by adding a stabilization stiffness matrix to the stiffness $\mathbf{K}_{e}$. This method is drawn from the approach of Reference [20] in which an efficient stabilization technique and an assumed strain method were applied to the eight-node hexahedral element with uniform reduced integration. The stabilization forces are derived in the same way.

The starting point consists in decomposing the discrete gradient operator $\overline{\hat{\mathbf{B}}}$, defined by Eq. (14), into two parts as follows:

$$
\overline{\hat{\mathbf{B}}}=\hat{\mathbf{B}}_{12}+\overline{\hat{\mathbf{B}}}_{34}
$$

The first term in this additive decomposition is given by Eq. (15), while the second term $\overline{\hat{\mathbf{B}}}_{34}$ is precisely the one that vanishes at the integration points. The elastic stiffness is then given by Eq. (10) as the sum of the following two contributions:

$$
\begin{aligned}
\mathbf{K}_{12} & =\int_{\Omega_{e}} \hat{\mathbf{B}}_{12}^{T} \cdot \mathbf{C} \cdot \hat{\mathbf{B}}_{12} d \Omega=\sum_{J=1}^{n_{\text {int }}} \omega\left(\zeta_{J}\right) J\left(\zeta_{J}\right) \hat{\mathbf{B}}_{12}^{T}\left(\zeta_{J}\right) \cdot \mathbf{C} \cdot \hat{\mathbf{B}}_{12}\left(\zeta_{J}\right) \\
\mathbf{K}_{S T A B} & =\int_{\Omega_{e}} \hat{\mathbf{B}}_{12}^{T} \cdot \mathbf{C} \cdot \overline{\hat{\mathbf{B}}}_{34} d \Omega+\int_{\Omega_{e}} \overline{\hat{\mathbf{B}}}_{34}^{T} \cdot \mathbf{C} \cdot \hat{\mathbf{B}}_{12} d \Omega+\int_{\Omega_{e}} \overline{\hat{\mathbf{B}}}_{34}^{T} \cdot \mathbf{C} \cdot \overline{\hat{\mathbf{B}}}_{34} d \Omega
\end{aligned}
$$

The stabilization stiffness, Eq. (19), vanishes at the integration points and is hence calculated in a co-rotational coordinate system given in [20]. This orthogonal co-rotational system, which is embedded in the element and rotates with it, is chosen to be aligned with the referential coordinate system. This choice of a co-rotational approach has numerous advantages, including simplified expressions for the above stabilization stiffness matrix, whose first two terms vanish, and a more effective treatment of shear locking in this frame.

In the same way, the internal forces of the element can be written as:

$$
\mathbf{f}^{\text {int }}=\mathbf{f}_{12}^{\text {int }}+\mathbf{f}^{S T A B}
$$

The first term, $\mathbf{f}_{12}^{\text {int }}$, is the only one taken into consideration when the forces are evaluated at the integration points:

$$
\mathbf{f}_{12}^{\text {int }}=\int_{\Omega_{e}} \hat{\mathbf{B}}_{12}^{T} \cdot \boldsymbol{\sigma} d \Omega=\sum_{J=1}^{n_{\mathrm{int}}} \omega\left(\zeta_{J}\right) J\left(\zeta_{J}\right) \hat{\mathbf{B}}_{12}^{T}\left(\zeta_{J}\right) \cdot \boldsymbol{\sigma}\left(\zeta_{J}\right)
$$


The second term $\mathbf{f}^{\text {STAB }}$ of Eq. (20) represents the stabilization forces which are consistently calculated in the co-rotational coordinate system according to the stabilization stiffness matrix given by Eq. (19), see Reference [20] for further details. It is worth noting that, in the recently developed SHB8PS formulation (see Reference [19]), two integration points are sufficient for both providing a rank sufficient element and dealing with elastic problems. Therefore, throughout the numerical examples given in Section 3, only two Gauss integration points are considered.

\subsection{Limit-point buckling and Riks' modified method}

Buckling is a typical structural instability phenomenon, characterized by a sudden deflection of a (thin) structure when subjected to compressive loads. Such failure modes occur when the compressive load reaches a critical value. In practice, this phenomenon involves significant changes in the shape of the structure along with geometric nonlinear effects. Besides the well-known sensitivity of buckling to geometric imperfections, it is also known to be very sensitive to boundary conditions. Over the last decades, considerable effort has been devoted to the detection of such singular points (limit points or bifurcation points) and the associated post-buckling behavior. Various criteria and efficient algorithms have been developed to deal with this issue as demonstrated by the comprehensive literature in this field (Timoshenko and Gere [23], Koiter [24], Hutchinson and Koiter [25], Thompson and Hunt [26], Budiansky [27]).

Limit-point buckling is the type of buckling involving a sudden large movement (jumping) in the direction of the loading, as opposed to bifurcation buckling where the bifurcated branch intersects the fundamental path (branching), see Fig. 2. In this paper attention is confined to limit-point buckling; therefore we restrict ourselves to benchmark problems in this category. The modified path-following Riks method, which is the algorithm implemented in Abaqus for solving this type of problem [28], is the method used to obtain the load-deflection curves including the post-buckling response (see, e.g., Riks [29], Crisfield [30], Ramm [31]). In order to capture the complex load-displacement response, which can exhibit a decrease in load and/or displacement as the solution evolves, the equilibrium path is computed by including the load magnitude as an additional unknown in the formulation of the problem. This results in proportional loading as all load magnitudes then vary with a single scalar parameter, called the load proportionality factor. This method is also called an arc-length method because the equilibrium path in the new space, defined by the nodal variables and the loading parameter, is determined by using this so-called arc-length. The arc-length itself multiplies the load by a load factor, allowing both the load and the displacement to vary (i.e. to increase or decrease) throughout the time step. 


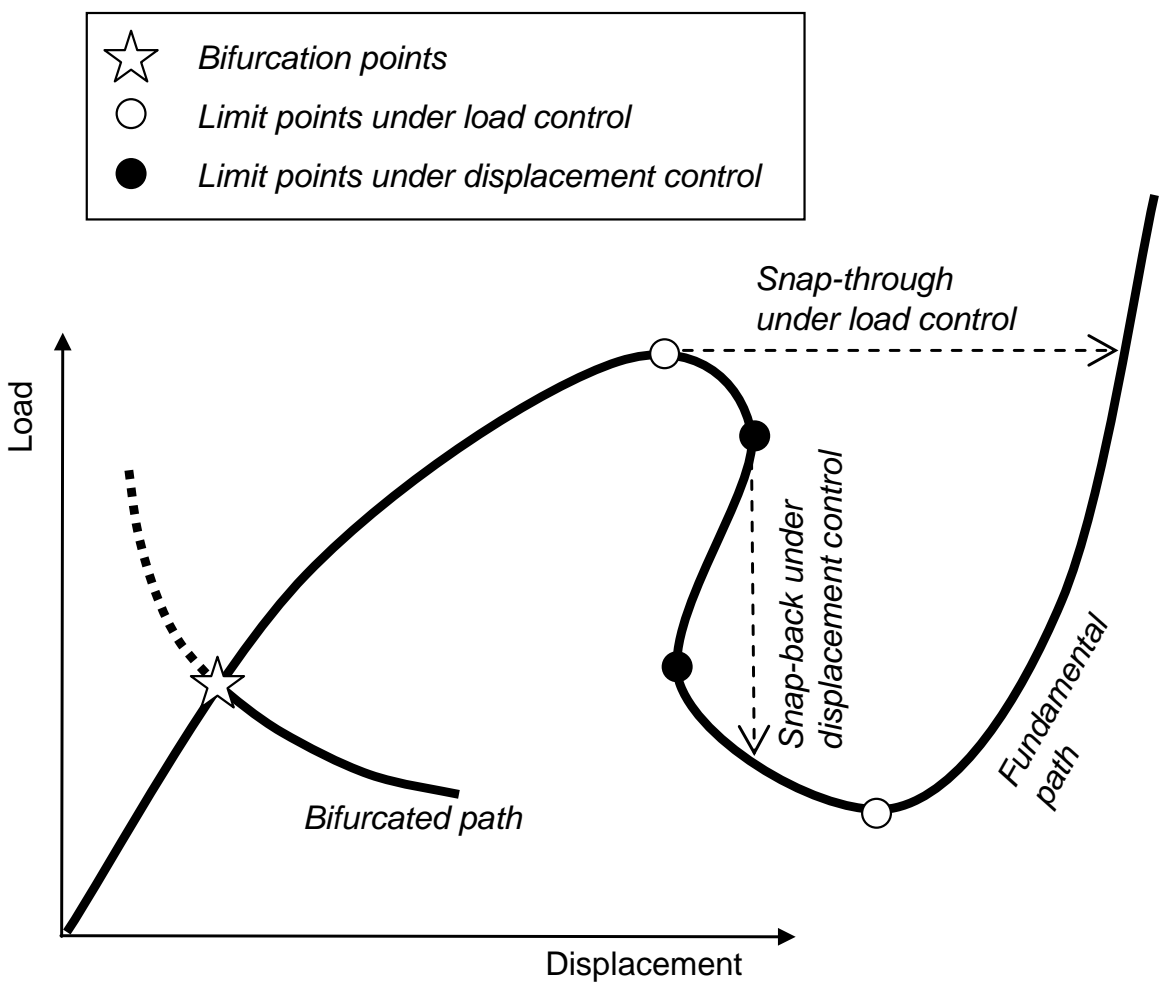

Fig. 2. Schematic representation of bifurcation and limit points.

Due to the nature of this technique, the loading applied to the structure is only used as an indication of the direction of loading. The actual load applied in the first increment is the product of this "reference" load and the initial arc-length, which is one of the inputs of this procedure. The arc-length is subsequently increased or decreased, depending on the rate of convergence of the equilibrium algorithm. The analysis is terminated when it reaches either an imposed node displacement or, most often, an imposed load value. In certain cases, the end point of the load-displacement curve will surpass the imposed stopping criterion, due to the arbitrary size of the arc-length when the last increment occurs. This may be seen on some of the load-displacement curves in the next section.

\section{Performance of SHB8PS in regards to limit-point buckling benchmark tests}

Six benchmark tests are used to investigate the relative ability of the SHB8PS element to predict limit-point buckling. The studied geometries are deep or shallow arches provided with thick or thin cylindrical sections. Concentrated and inclined loads are applied, together with clamped and hinged boundary conditions. These problems feature nonlinear 
pre-buckling as well as unstable buckling behavior. The material behavior is considered isotropic, linearly elastic for the six test problems.

The developed element is compared to the Abaqus linear shell element S4R5; this should be the most appropriate Abaqus linear element for the selected tests as shown in a recent study by Leahu-Aluas et al. [32]. Then, one of the most accurate Abaqus linear solid elements, denoted C3D8I, is also used for comparison purposes, since it shares with the SHB8PS element the same three-dimensional geometry. Finally, the solid-shell element SC8R is also investigated. It is noteworthy that the latter element has been only recently introduced in this commercial software and it has not been specifically designed for buckling applications. The main features of the Abaqus elements used for comparison are summarized in Table 1. The SHB8PS characteristics have been briefly described in Section 2: this is an eight-node hexahedron with three displacement degrees of freedom per node and it is provided with a physical stabilization scheme. Also, the SHB8PS employs $1 \times n_{\text {int }}$ integration points (i.e., one single in-plane integration point, and $n_{\text {int }}$ points through the thickness). As already mentioned, only two Gauss integration points will be considered for the subsequent elastic benchmark problems. 


\section{Table 1}

Main features of the S4R5 (linear shell), C3D8I (linear solid) and SC8R (linear solid-shell) elements.

\begin{tabular}{cccc}
\hline & S4R5 & C3D8I & SC8R \\
\hline Number of nodes & 4 & 8 & 8 \\
Integration points & $1 \times n^{*}$ & 8 & $1 \times n^{*}$ \\
Degrees of freedom & 5 (3 displ., 2 rot.) & 3 (displacements) & 3 (displacements) \\
Hourglass treatment & Not applicable & Not applicable & Default stabilization \\
Applicable strain & Small & Finite & Finite \\
Intended thickness & Thin only & Thin/thick & Thin/thick \\
\hline Intrary number $(n)$ of through-thickness integration points can be used (the default value is five).
\end{tabular}

The reference solutions are available in the literature for all of the selected problems. Moreover, for most of these test problems the reference solutions have been previously validated and updated in [32].

The nomenclature given below is employed throughout this article.

\section{$\underline{\text { Nomenclature and naming conventions }}$}

u Displacement length (l) Longest side of the structure

$\mathrm{R}$ Radius width (w) Side perpendicular to the loading direction

$\mathrm{P}$ Applied load thickness (t) Side parallel to the loading direction

E Elasticity modulus

$v$ Poisson's ratio The mesh naming convention is length $\times$ width $(\times$ thickness)

I Area moment of inertia

\subsection{Clamped deep circular arch subjected to a concentrated load}

This test was treated numerically by Wriggers and Simo [33], Jiang and Chernuka [34] and Domissy [1]. The geometry, boundary conditions and loading for this test are sketched in Fig. 3. It consists of a deep circular arch fixed at both ends and subjected to a concentrated force at the apex. Since the force, geometry and boundary conditions are symmetric, only half of the structure is modeled for simulation. 


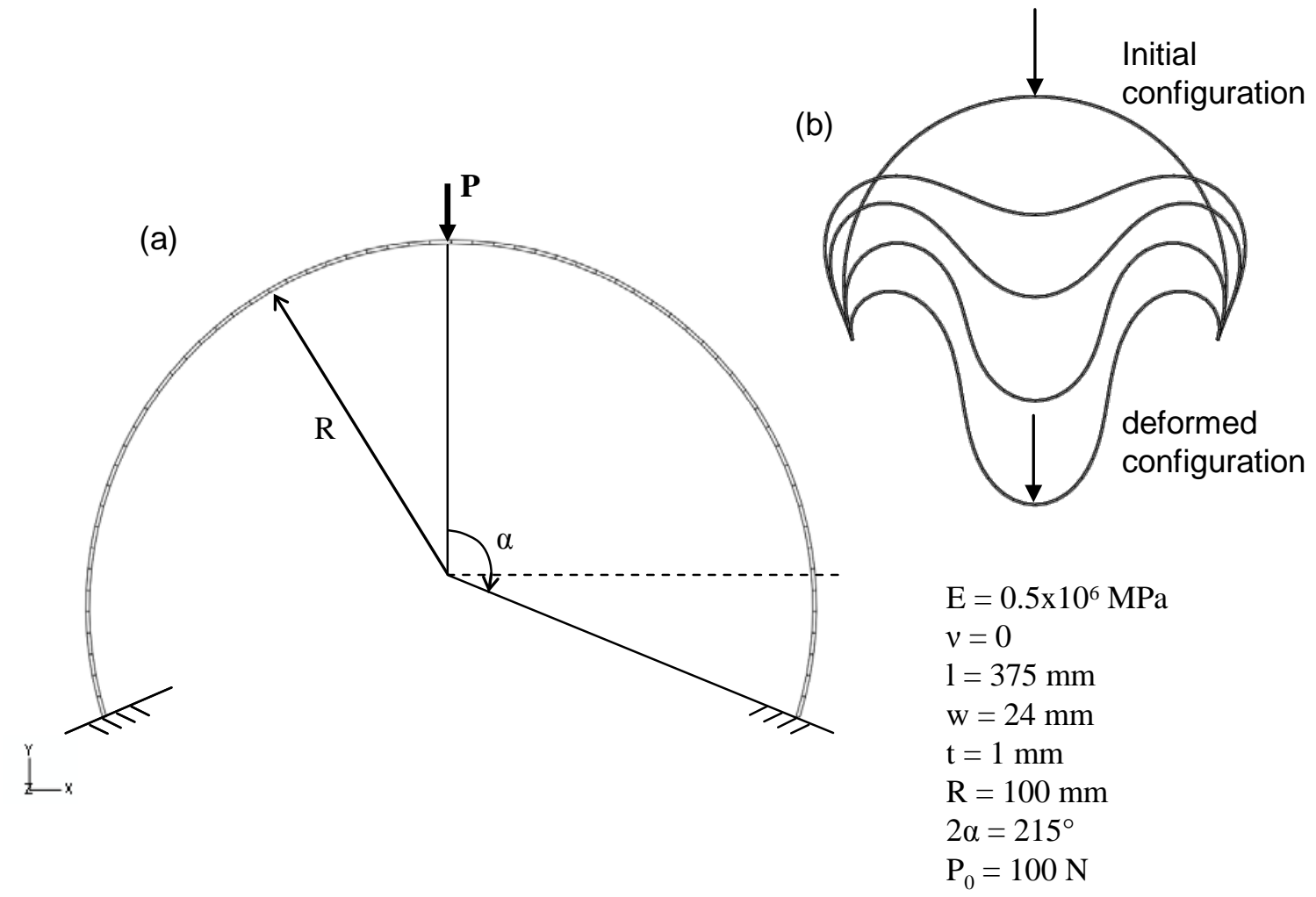

Fig. 3. (a) Geometry for the clamped deep circular arch subjected to a concentrated load; and (b) initial and successive deformed configurations.

The results for the four selected elements are shown in Fig. 4. The convergence of the elements SHB8PS, C3D8I, S4R5, and SC8R is compared in this figure. The mesh sizes are given in the following order: length $\times$ width $\times$ thickness. All benchmark tests in Section 3 include only elastic deformation for the given structures. 

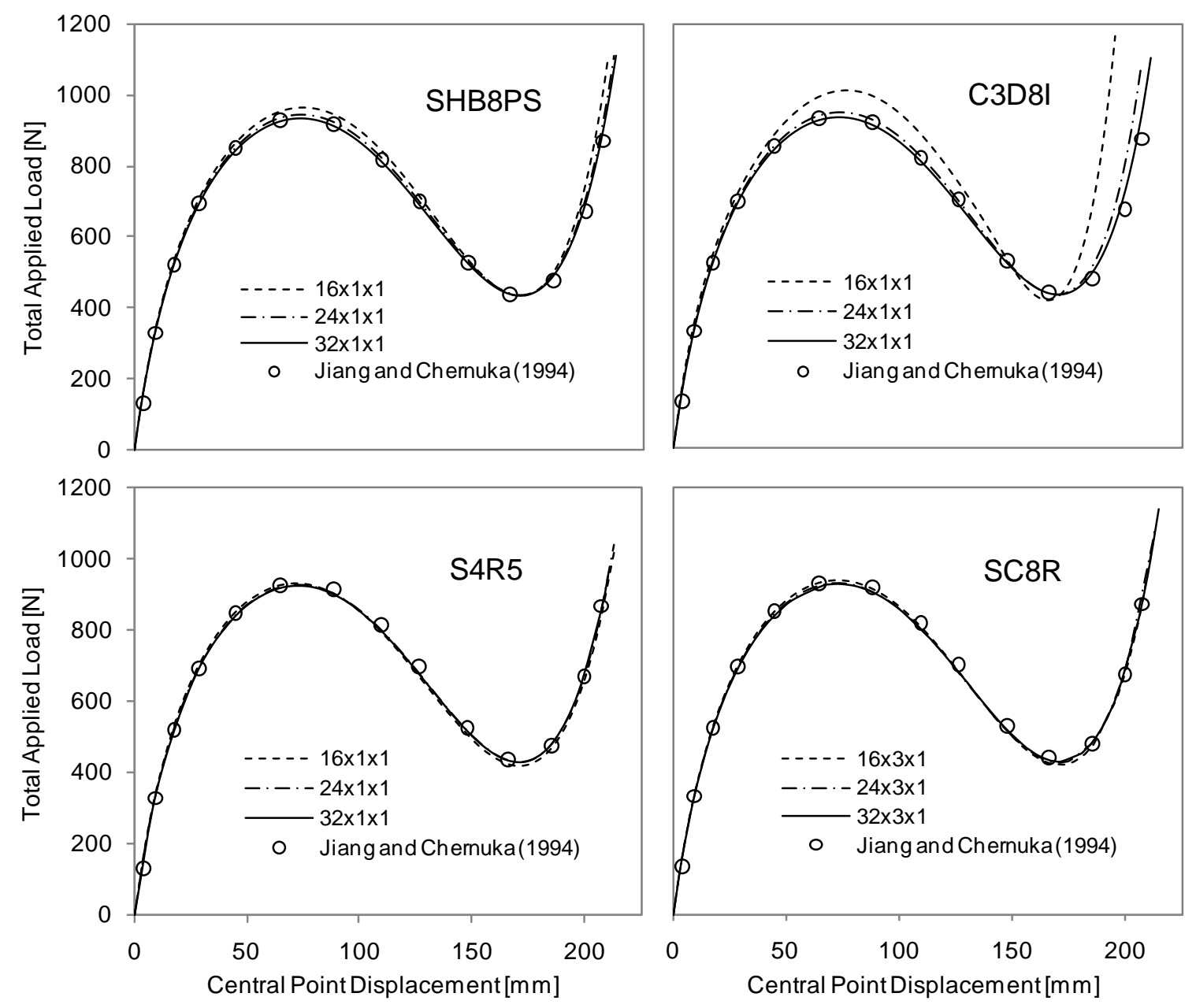

Fig. 4. Load-displacement curves for the clamped deep circular arch subjected to a concentrated load.

It is clear from Fig. 4 that the SHB8PS element outperforms the solid element C3D8I. It appears nevertheless that the SC8R element performs better than SHB8PS. Note that the number of elements in the width direction for the SC8R element is larger than for the other elements. While most elements require only one element in the width direction in order to converge towards the reference solution, the behavior of the SC8R element seems to be different. Fig. 5 examines the effect of the number of elements in the width direction for the SC8R element. It appears from this figure that the solution somehow deviates whenever less than three elements are used in the width direction. In addition, zero displacements in the $z$ direction had to be imposed in order to obtain a solution with only one SC8R element in the width direction. This suggests that the SC8R element, recently introduced in Abaqus, has not been designed to optimally handle this particular type of problem. Thus it is used in this paper only for illustration purposes due to the solid-shell nature of its formulation. 

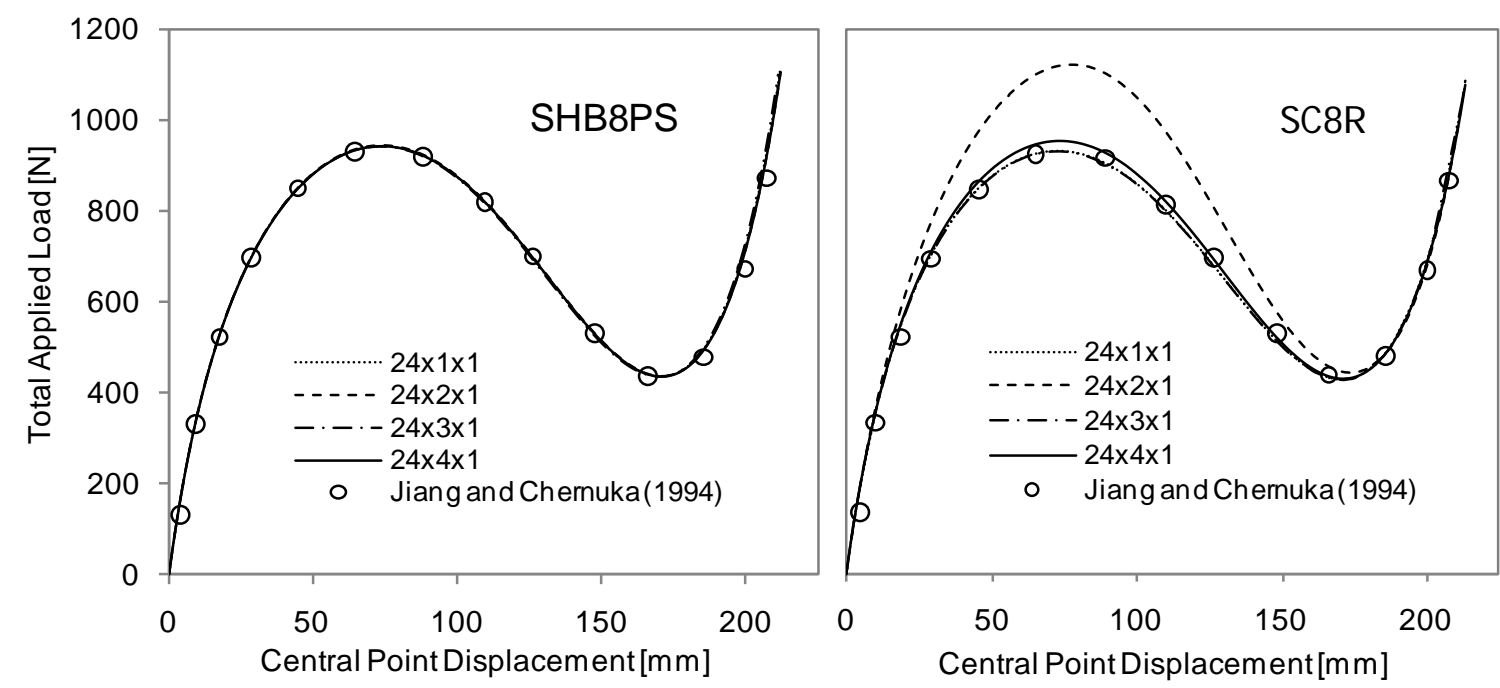

Fig. 5. Solution curves showing the behavior of the SC8R element compared to the SHB8PS as the number of elements in the width of the structure is varied.

\subsection{Hinged deep circular arch subjected to a concentrated load}

The geometry for this test can be found in Fig. 6. This test was treated by Wriggers and Simo [33] and by Boutyour et al. [35] who provided a numerical solution in terms of the load proportionality factor versus displacement. This test is similar to the preceding one except for the change in boundary conditions. Symmetry was again used since it is the symmetric limit-point buckling response that is of interest in the current analysis. Fig. 7 shows the results obtained for this test. 


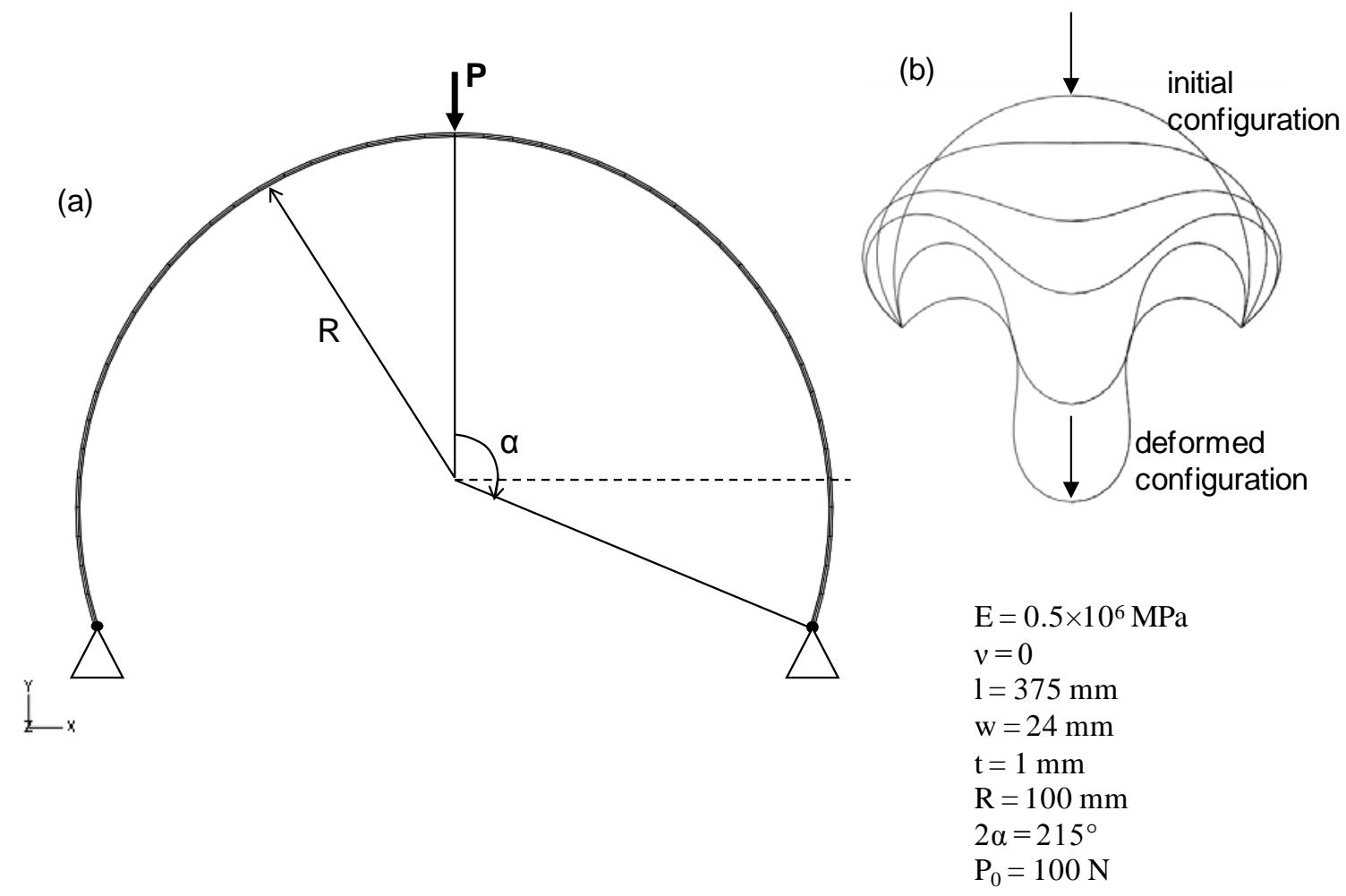

Fig. 6. (a) Geometry for the hinged deep circular arch subjected to a concentrated load; and (b) initial and successive deformed configurations during loading. 

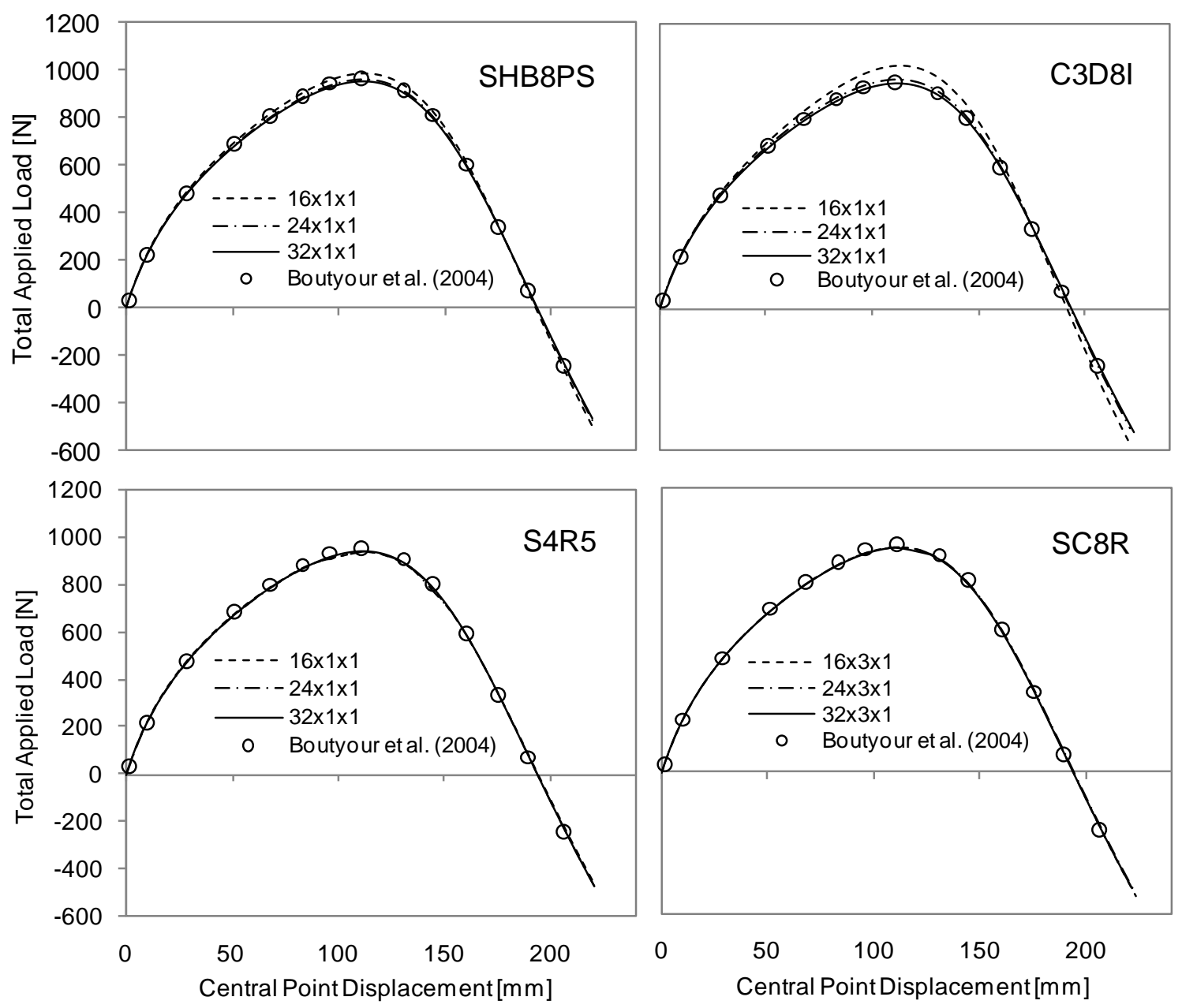

Fig. 7. Load-displacement curves for the hinged deep circular arch subjected to a concentrated load.

The conclusions are very similar to those of the previous test. While all the elements converge to the same solution, the solid element proves less appropriate to describe this thin structure with coarse meshes. The convergence of the SHB8PS element is successful and compares to that of the S4R5 shell and SC8R solid-shell elements. As in the previous benchmark test, it can be seen from Fig. 8 that the SC8R required more elements in the width in order to converge. This issue will not be commented hereafter since it is common to all the subsequent benchmark problems and, if carefully treated, the response of the SC8R element proves very accurate and effective. 

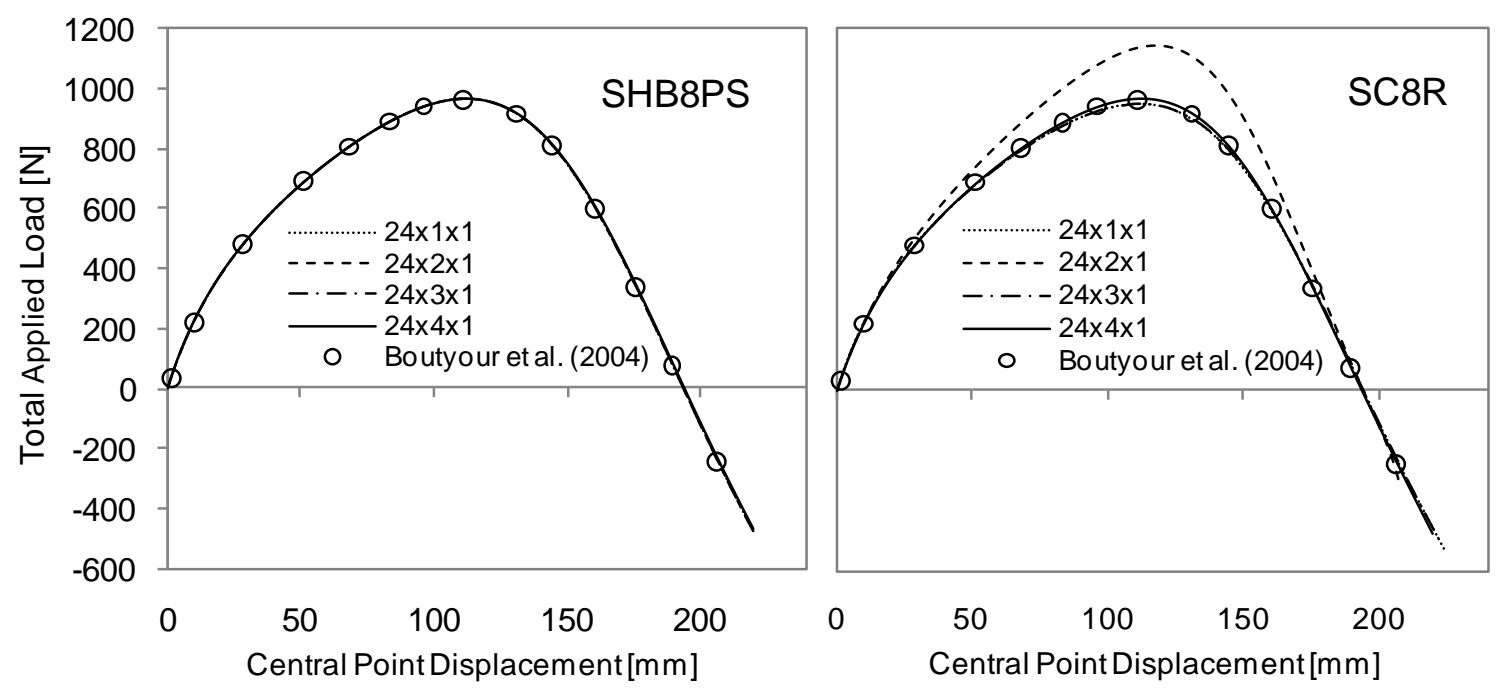

Fig. 8. Hinged deep circular arch: sensitivity to the mesh density in the width direction for the SC8R element.

\subsection{Clamped-hinged deep circular arch subjected to a concentrated load}

This test was treated numerically by Surana [36], Simo and Vu-Quoc [37], Jiang and Chernuka [34], among others. DaDeppo and Schmidt [38] provided the analytical solution for this kind of problem and it is also considered in the Abaqus manual [28]. The geometry for this test is provided in Fig. 9 together with the material data and the deformed shape of the structure. Due to the non-symmetry of boundary conditions, the entire structure was modeled. Results for this benchmark test include two different load-displacement curves, for the $x$ and $y$ directions. The results for this test can be found in Fig. 10 and Fig. 11, respectively. It is clear again that the SHB8PS is more accurate than the C3D8I, whenever the mesh becomes coarse. The S4R5 shell element shows very good results, and also the SC8R solid-shell element exhibits a good convergence rate for this test. 

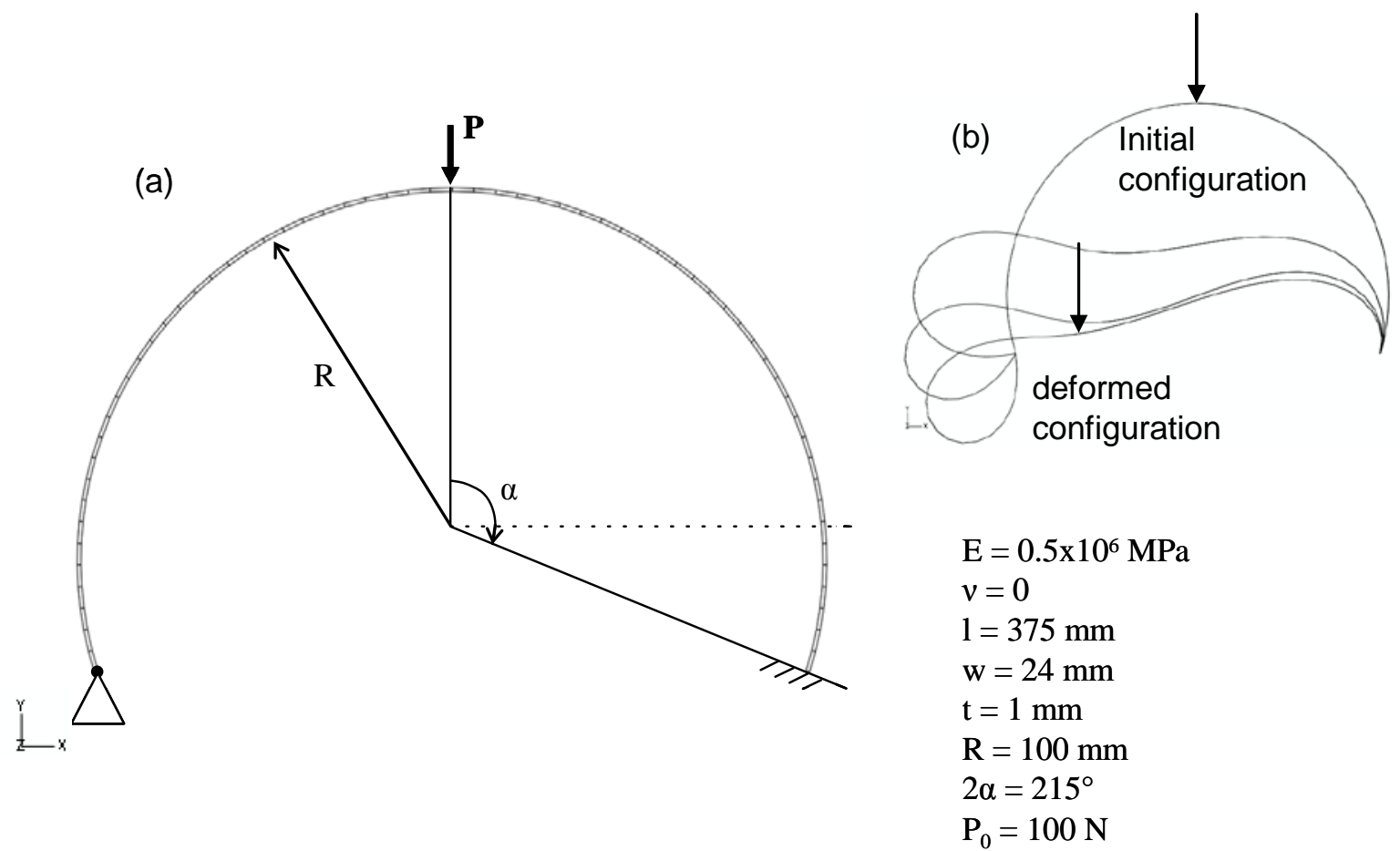

Fig. 9. (a) Geometry for the clamped-hinged deep circular arch subjected to a concentrated load; and (b) initial and successive deformed configurations during loading. 

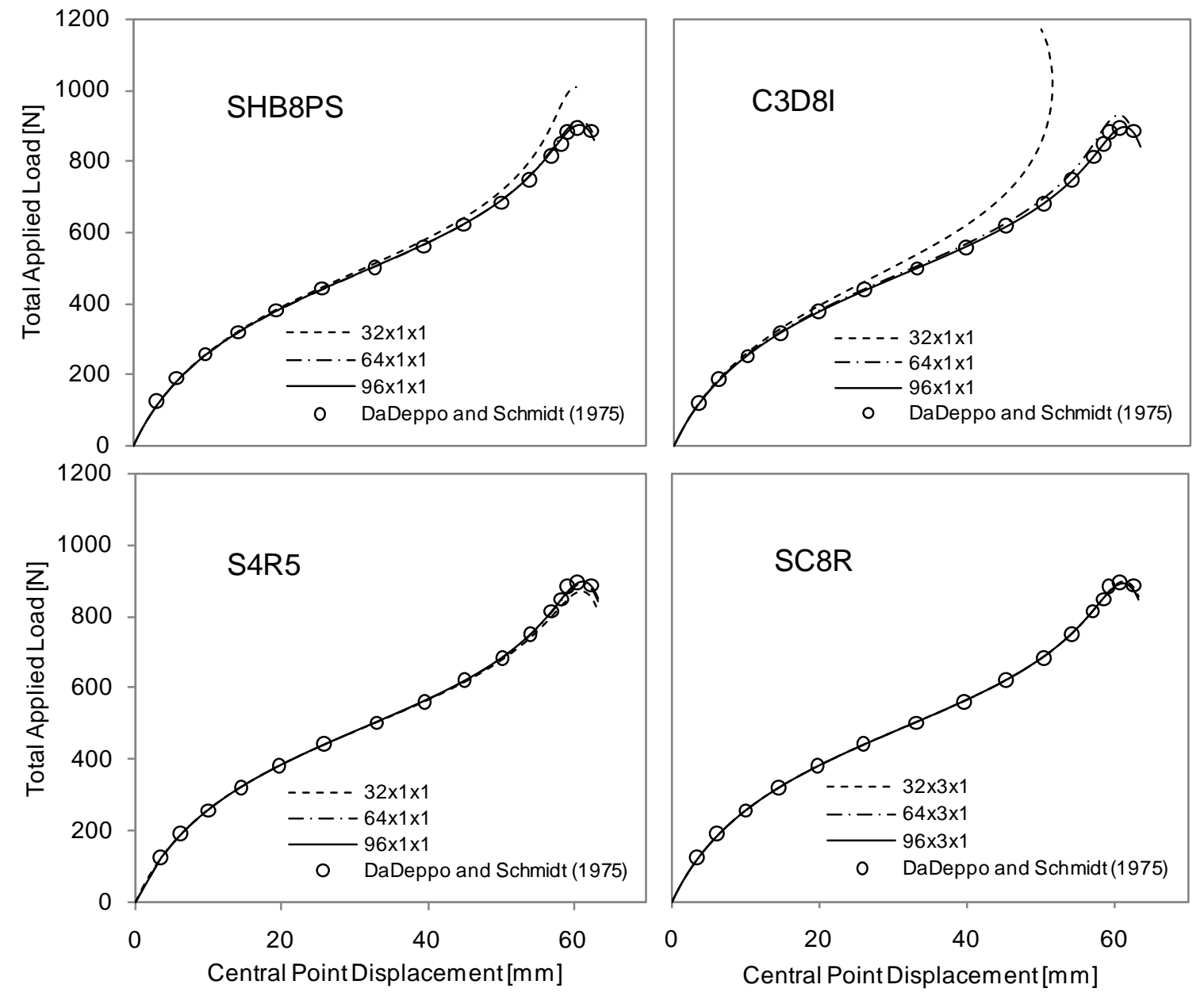

Fig. 10. Load-displacement curves in the $x$ direction for the clamped-hinged deep circular arch subjected to a concentrated load. 

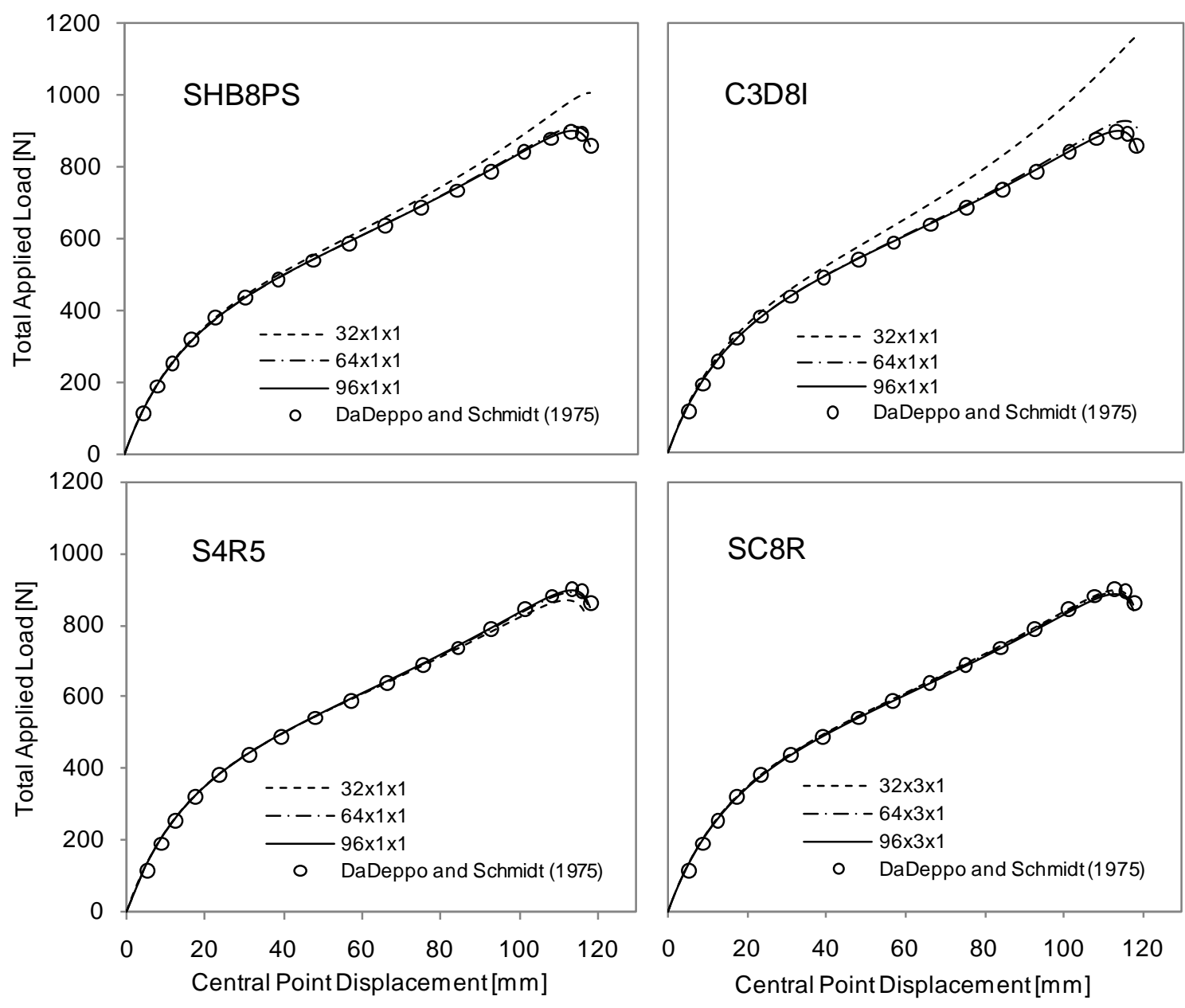

Fig. 11. Load-displacement curves in the $y$ direction for the clamped-hinged deep circular arch subjected to a concentrated load.

\subsection{Hinged shallow circular arch subjected to an inclined load}

This test was presented by Kim and Kim [39] who solved numerically for the response. The geometry and loading are shown in Fig. 12 along with the material properties. The entire structure was modeled due to the non-symmetric nature of the applied load which is what makes this test different from the other benchmark tests. In addition, in order to obtain an accurate result for the circumferential displacement (in the $x$-direction), it was necessary to keep at least two elements in the width of the structure. This allowed for accurate modeling of the inclined force without having to approximate the load by dividing it in half and applying it to the edges of the structure. The Abaqus continuum shell element SC8R would not converge for this test. The solutions for the remaining elements are shown in Fig. 13 and Fig. 14. Compared to the shell element, the SHB8PS and C3D8I elements converge in a similar way, with SHB8PS being more accurate in the limit-point 
zone. It is noteworthy that the correct response prediction for this structure required several elements in the width direction for all elements (at least four elements for the S4R5). Also, two elements were required in the thickness direction for the C3D8I and SHB8PS elements. Indeed, this test shows sensitivity to the location of the hinged boundary condition at the edges of the structure. This aspect will be further investigated and clarified in Section 4.

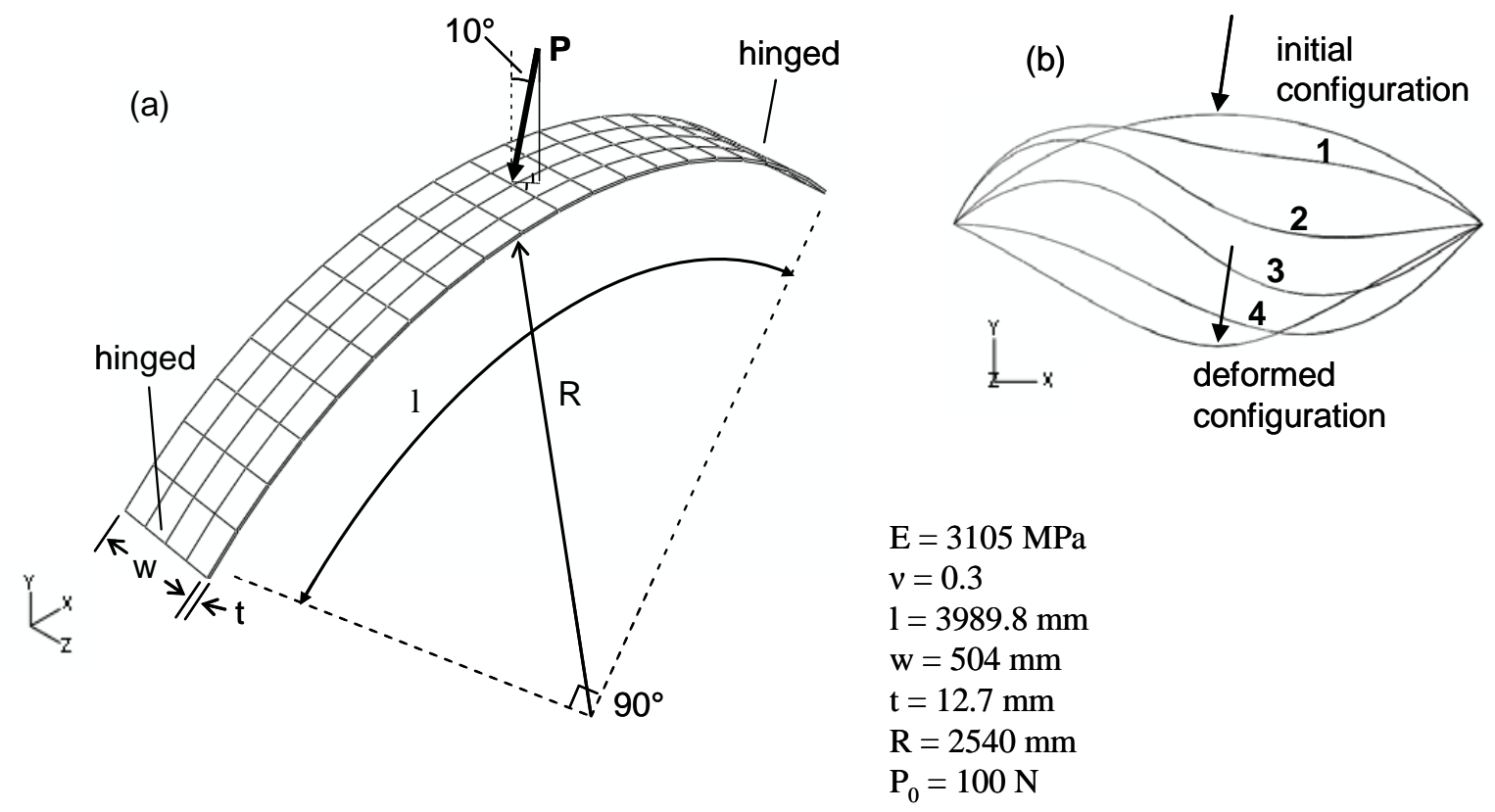

Fig. 12. (a) Geometry for the hinged shallow circular arch subjected to an inclined load; and (b) initial configuration and subsequent deformation of the structure. 

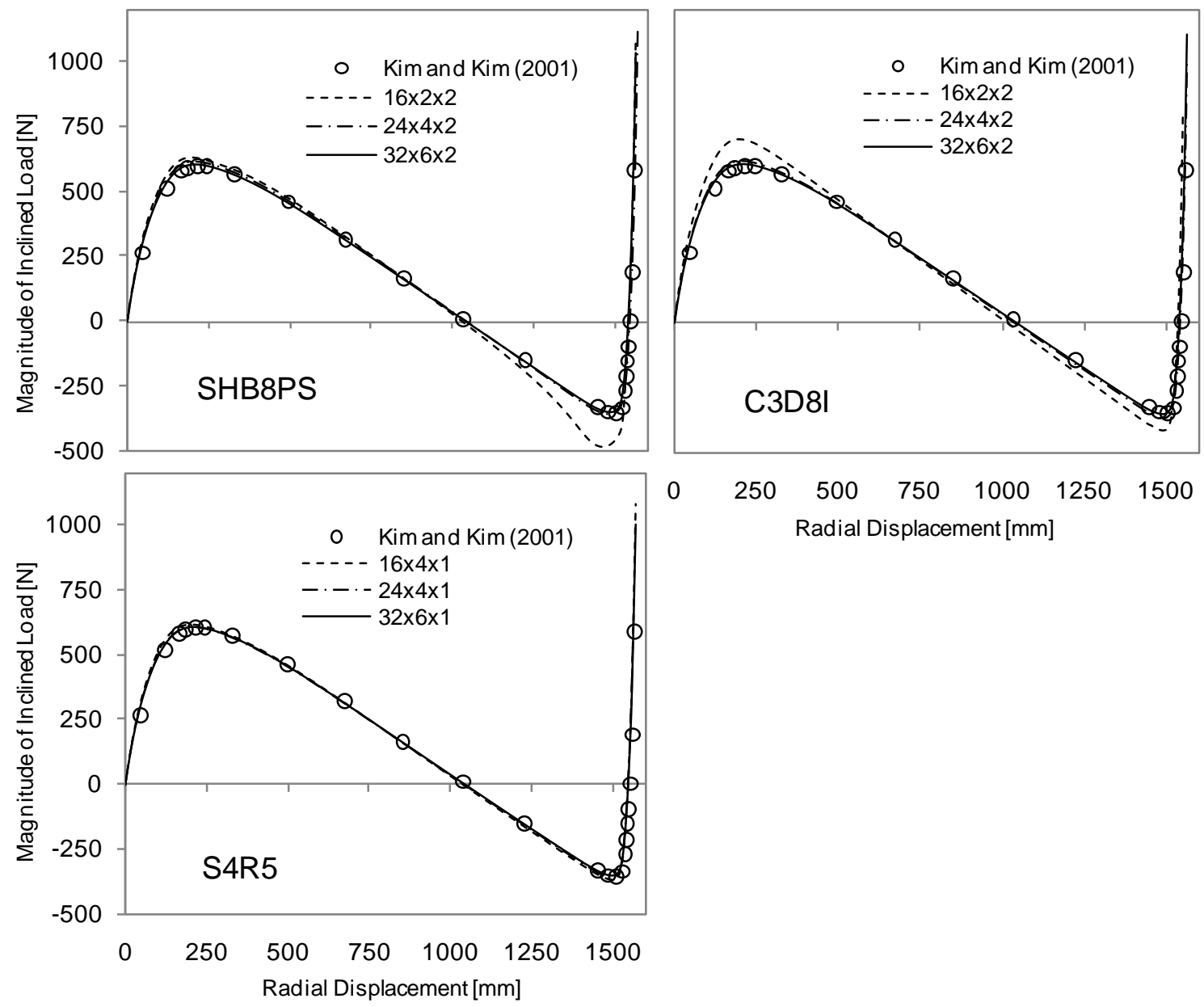

Fig. 13. Load-displacement curves for the hinged shallow circular arch subjected to an inclined load: Radial displacement. 


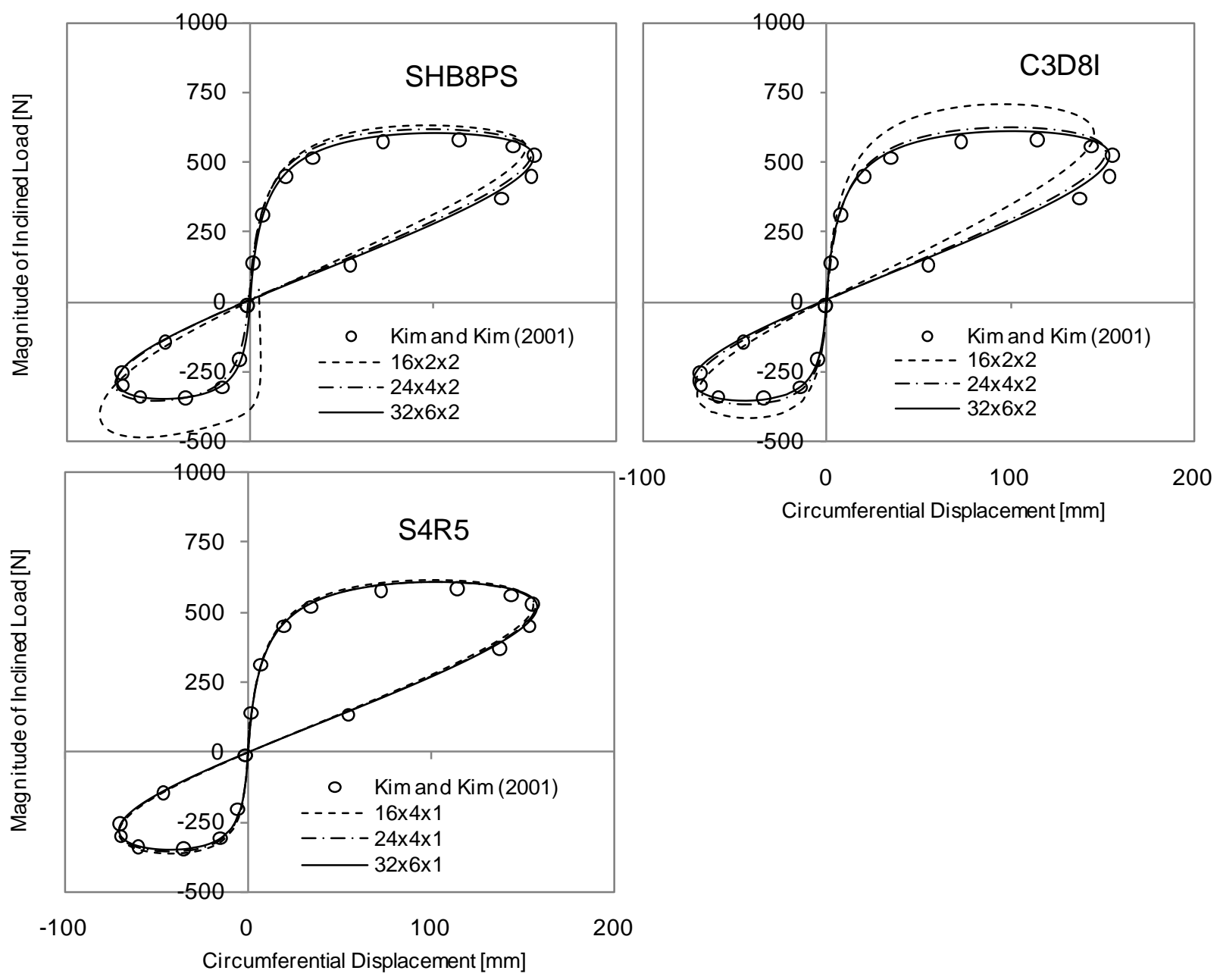

Fig. 14. Load-displacement curves for the hinged shallow circular arch subjected to an inclined load: Circumferential displacement.

\subsection{Hinged thick cylindrical section subjected to a central concentrated load}

This benchmark test has been studied by several authors (e.g., Klinkel and Wagner [40], Sze and Zheng [41], Legay and Combescure [9], Sze et al. [42], Kim et al. [15]) who provided numerical solutions. Symmetry was exploited and hence only a quarter of the structure was modeled. The geometry is shown in Fig. 15 and the results are given in Fig. 16. Two reference solutions, from Klinkel and Wagner [40] and Sze et al. [42], are provided hereafter since they are slightly different. 

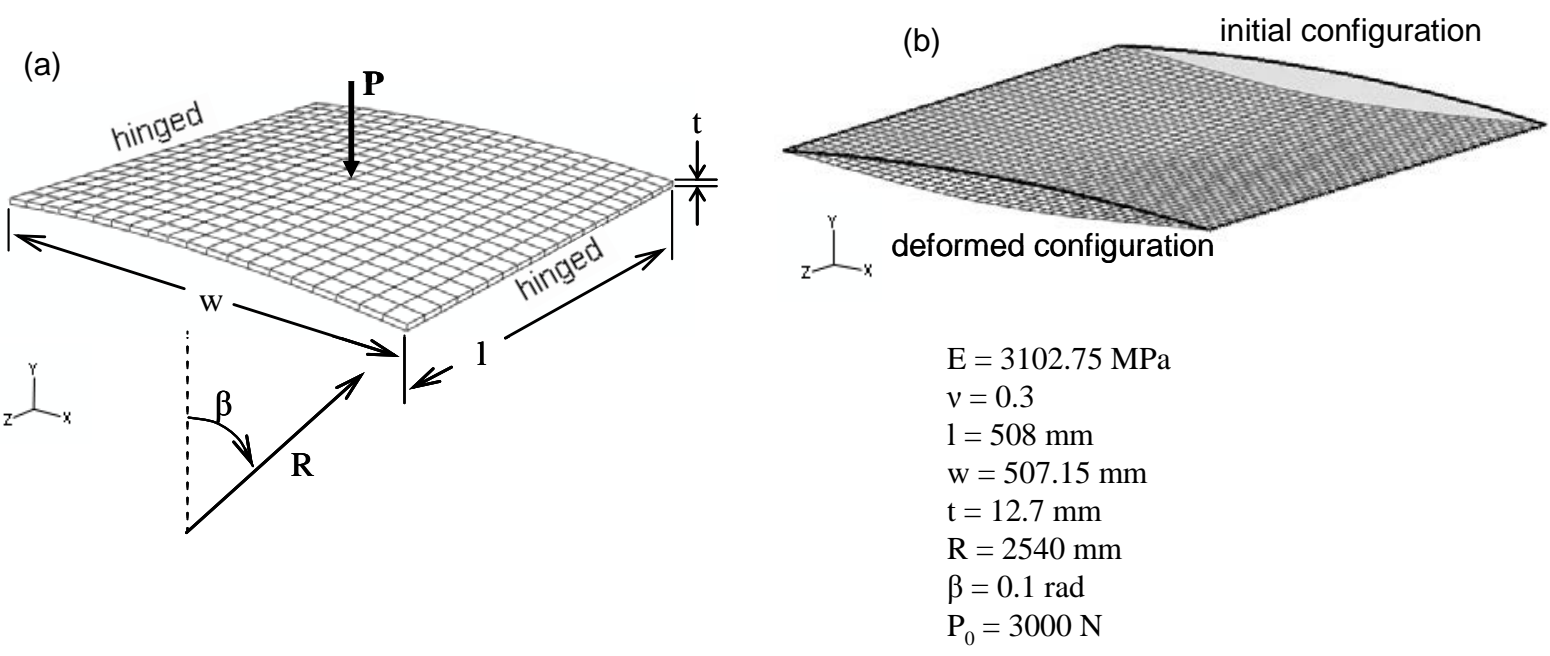

Fig. 15. (a) Geometry for the hinged thick cylindrical section subjected to a central concentrated load; and (b) initial and intermediate deformed configurations. 

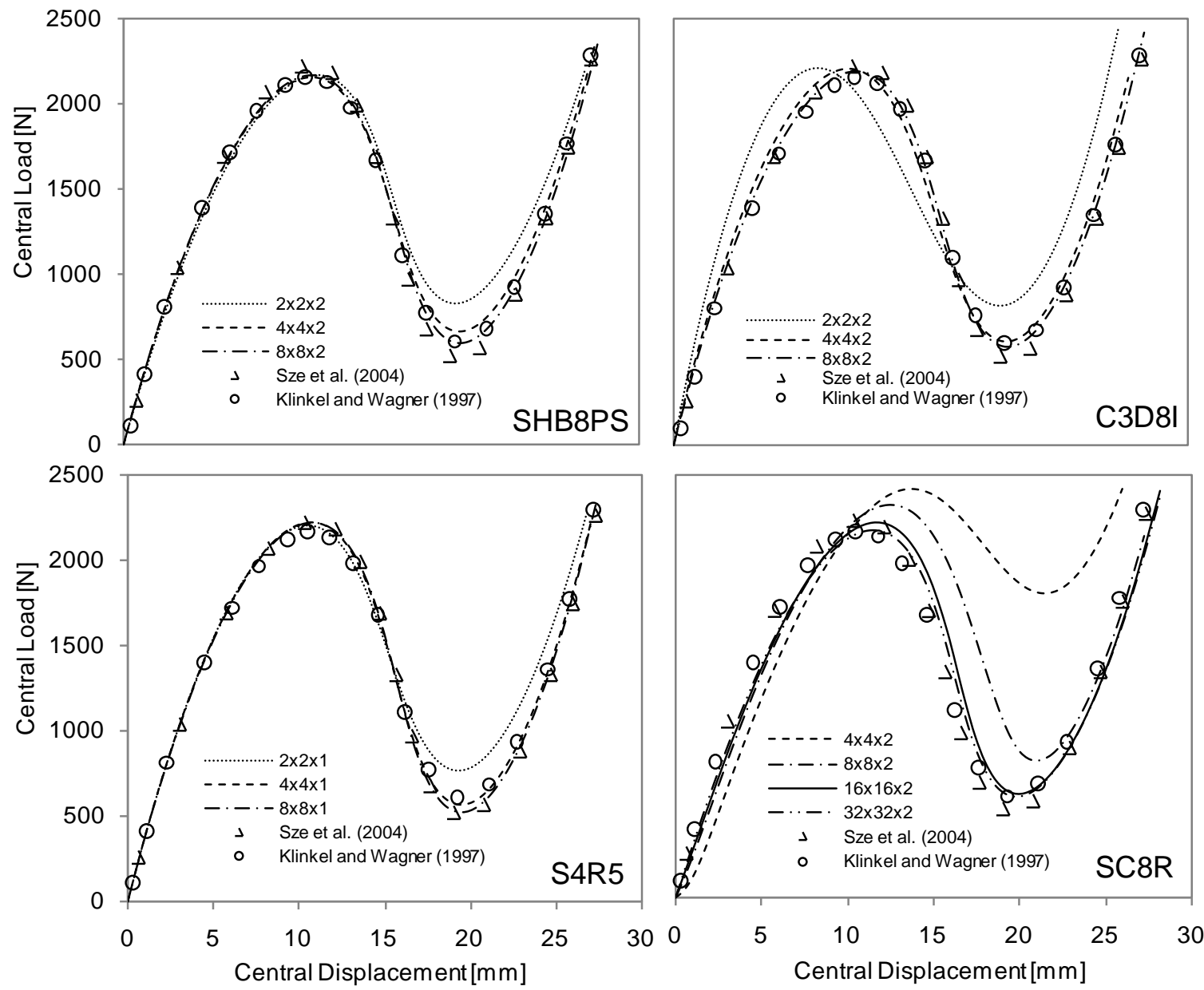

Fig. 16. Load-displacement curves for the hinged thick cylindrical section subjected to a central concentrated load.

Two noteworthy observations can be made. The first is that even for a coarse mesh of $2 \times 2 \times 2$ elements, the SHB8PS converged quite well; the limit-point location is accurately predicted with this coarse mesh, in contrast to the C3D8I solid element. The convergence rate of SHB8PS closely follows that of the shell element S4R5. This as well as many of the previous tests show its robustness when the aspect ratio is not maintained close to unity. The second observation is that the SC8R element exhibits a slower convergence rate on this test, as compared to the SHB8PS element. 


\subsection{Hinged thin cylindrical section subjected to a central concentrated load}

This test is the same as the previous one except for its cross-section which is now half as thick. This popular test was studied by numerous authors (e.g., Crisfield [30], Ramm [31], Kim and Kim [39], Sze and Zheng [41], Sze et al. [42], Boutyour et al. [35], Alves de Sousa et al. [17]) who solved for only numerical solutions. The geometry can be seen again in Fig. 17 and by virtue of symmetry only a quarter was modeled for the calculations. The results for this thin cylindrical section are shown in Fig. 18.

(b)
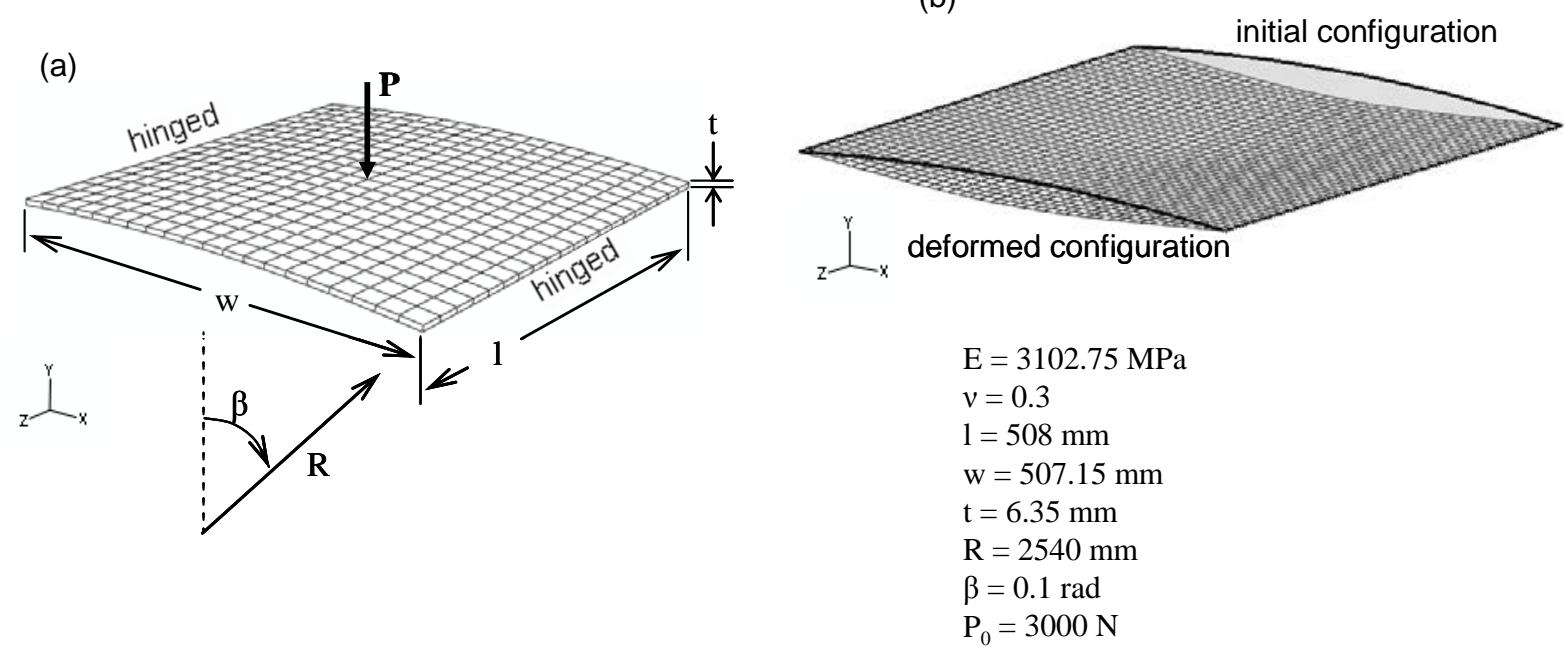

Fig. 17. (a) Geometry for the hinged thin cylindrical section subjected to a central concentrated load; and (b) initial and intermediate deformed configurations. 


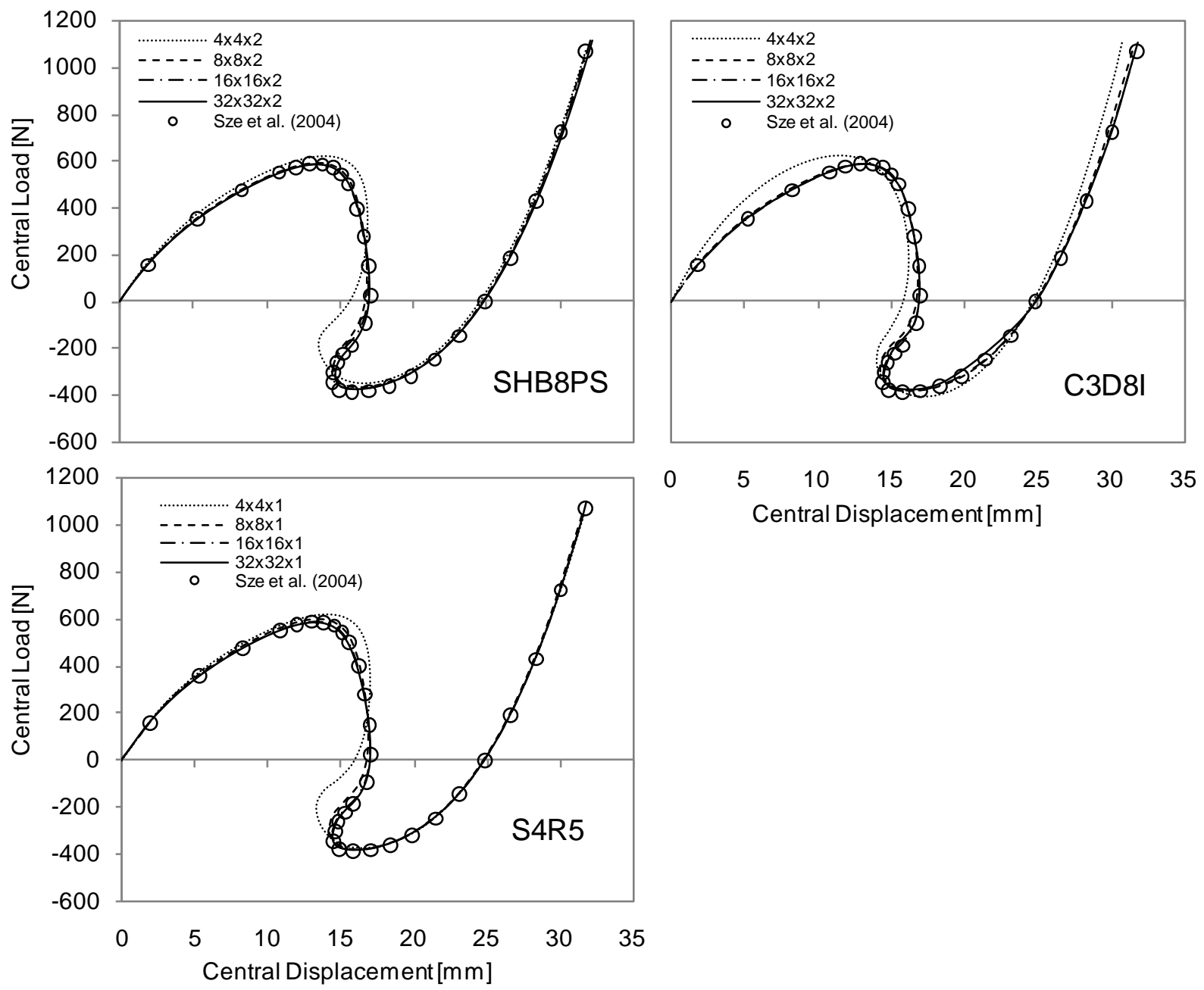

Fig. 18. Load-displacement curves for the hinged thin cylindrical section subjected to a central concentrated load.

Again, the convergence rates and predictions of the SHB8PS solid-shell and S45R shell elements are very similar. However, in addition to snap-through, this test also exhibits the particular feature of snap-back as compared to the previous benchmark problems. For this test, the SC8R would only converge for very fine meshes.

\section{Simulation of varied boundary conditions with SHB8PS for thin structures}

Thin structures are classically modeled by shell elements. A consequence of this modeling choice is that the boundary conditions are implicitly applied to the middle layer of the structure. Most of the time, this assumption is sufficient. However, real boundary conditions are often applied to an edge of the structure, rather than to its middle layer. Whenever the displacements become large, this may play a role in the response of the 
structure. The occurrence of buckling is a particular case when the sensitivity of the response to the loading details is exacerbated. It is noteworthy that with solid-shell (or solid) elements, the boundary conditions can naturally be applied to the edges of the structure (upper, lower, both). Two layers of elements may be used whenever a boundary condition must be applied to the middle surface. This also allows for a rigorous comparison with shell elements.

The aim of this section is to investigate the sensitivity of the previous tests with respect to the particular location of the boundary conditions at the ends of the structure. It also shows the ability of the SHB8PS element to accurately model boundary conditions other than those along the middle layer of a structure. For each test a converged mesh size was chosen for each element and only the location of the boundary conditions was varied. The last four tests from the previous section were selected, since they represent a variety of geometries and loading conditions:

- Clamped-hinged deep circular arch subjected to a concentrated load (see Section 3.3);

- Hinged shallow circular arch subjected to an inclined load (see Section 3.4);

- Hinged thick cylindrical section subjected to a central concentrated load (see Section 3.5);

- Hinged thin cylindrical section subjected to a central concentrated load (see Section 3.6).

The investigation revealed that the first two benchmark tests show low sensitivity to the location of the boundary conditions, while the two other tests are dramatically sensitive and require particular attention.

\subsection{Clamped-hinged deep circular arch subjected to a concentrated load}

The geometry and loading for this test are depicted in Fig. 9. The hinged boundary condition was varied here using both the C3D8I and SHB8PS elements. Both elements were used in order to validate any solution that was obtained. The boundary condition was placed on the upper and lower edges of the same surface where the original boundary condition was imposed. The results obtained for these various tests are shown in Fig. 19. The results for the various boundary conditions were very similar to each other. Therefore, only one element has been considered in the thickness for the corresponding benchmark test in Section 3.3. 

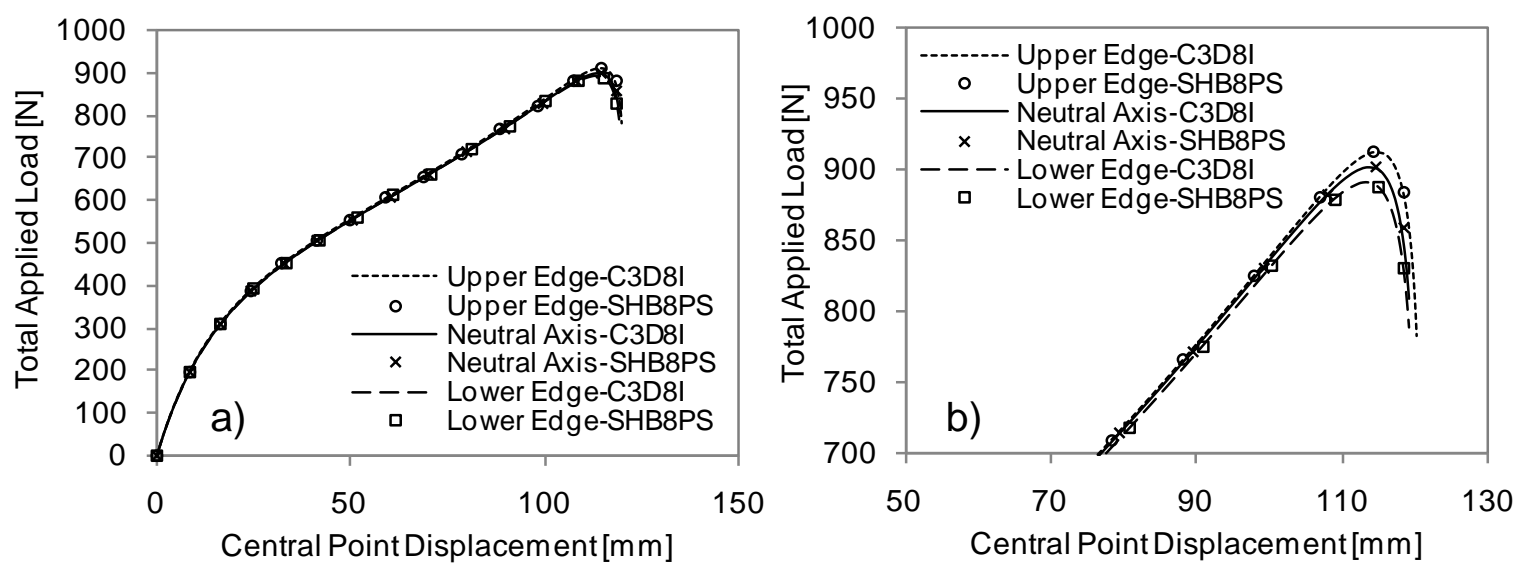

Fig. 19. (a) Load-displacement curves for various boundary conditions for the clampedhinged deep circular arch subjected to a concentrated load. (b) Close-up of solution curve affected by varying boundary conditions.

\subsection{Hinged shallow circular arch subjected to an inclined load}

This boundary condition test is for the geometry in Section 3.4 (Fig. 12). The hinged boundary condition was again placed on the upper edge, on the lower edge or in-between. The results obtained for these various tests are shown in Fig. 20.
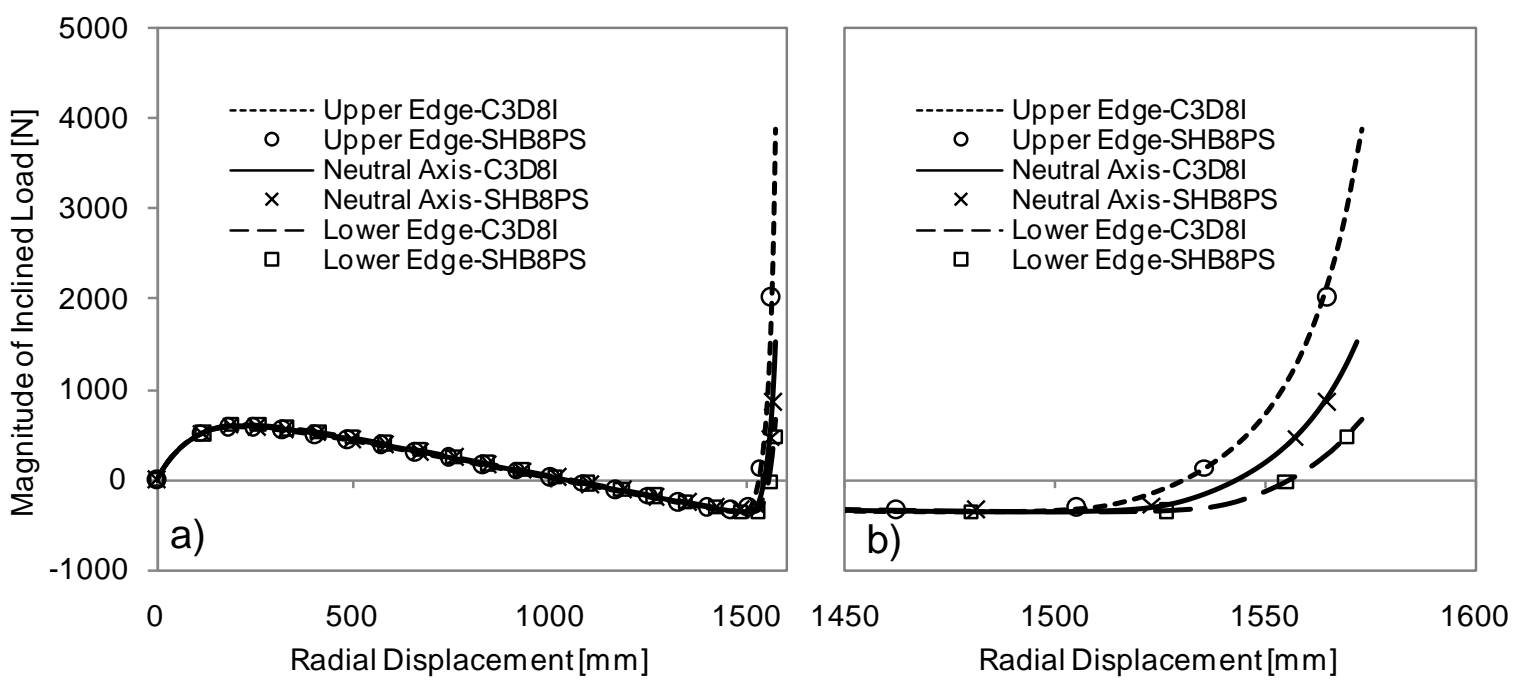

Fig. 20. (a) Load-displacement curves for various boundary conditions for the hinged shallow circular arch subjected to an inclined load. (b) Close-up of solution curve affected by varying boundary conditions. 
The sensitivity to a change in boundary conditions for this test is not particularly significant, although higher than for the previous test. Only in the last part of the curve, as the slope of the solution curve becomes very steep, rather different loads are recorded at the same displacement. However, in terms of benchmark tests, this test problem can still be successfully modeled with shell elements or with only a single solid or (solid-shell) element along the thickness.

\subsection{Hinged thick cylindrical section subjected to a central concentrated load}

This boundary condition test is for the geometry in Section 3.5 (Fig. 15). For the hinged boundary conditions, the same procedure as in the previous tests has been applied. The results are summarized in Fig. 21.

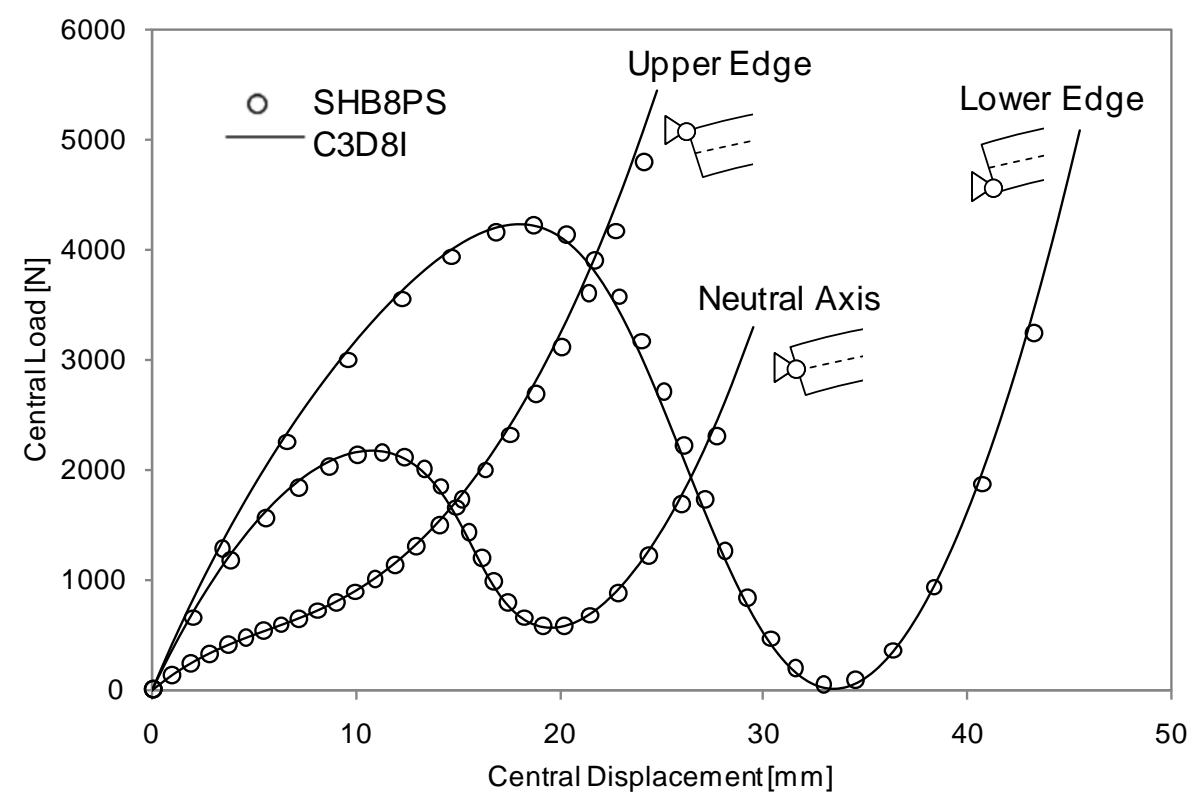

Fig. 21. Load-displacement curves for various boundary conditions for the hinged thick cylindrical section subjected to a concentrated load.

This test is particularly sensitive to changes in the boundary conditions; the snapthrough behavior is even precluded when the boundary conditions are prescribed at the upper edge, which highlights one of the benefits of the continuum shell formulation. Section 3 has shown that the SHB8PS was comparable or superior to the solid element C3D8I for the thin structure applications that were examined. In addition, the present test shows that it is also able to accurately model particular locations of the boundary conditions in the thickness direction, which is not possible with a shell element. Note that the SC8R 
was also tested in order to show how the Abaqus continuum shell element solution compared to the solution obtained with the SHB8PS; its response was comparable to the two other elements (SHB8PS solid-shell and C3D8I solid elements).

\subsection{Hinged thin cylindrical section subjected to a central concentrated load}

This boundary condition test is for the geometry in Section 3.6 (Fig. 17). The results (see Fig. 22) are similar to those for the hinged thick cylindrical section. The location of the boundary conditions dramatically influences the results. It is remarkable that the snapback phenomenon disappears when the hinged boundary conditions are applied to the upper or the lower edges, while it occurs for the mid-line condition. Also, the load and displacement values corresponding to the limit-point strongly vary from one case to the other. The SHB8PS and the C3D8I elements consistently give the same response in the three boundary condition situations. For such structures, the exact location of the boundary conditions with respect to the thickness does matter and hence needs to be precisely modeled. Whenever the boundary conditions are not prescribed at the middle surface, the use of shell elements would not be an adequate choice, and solid-shell elements provide a more reliable alternative instead. It is important to note that for most real-life structures, the mid-plane location of the boundary conditions represents only an approximation.

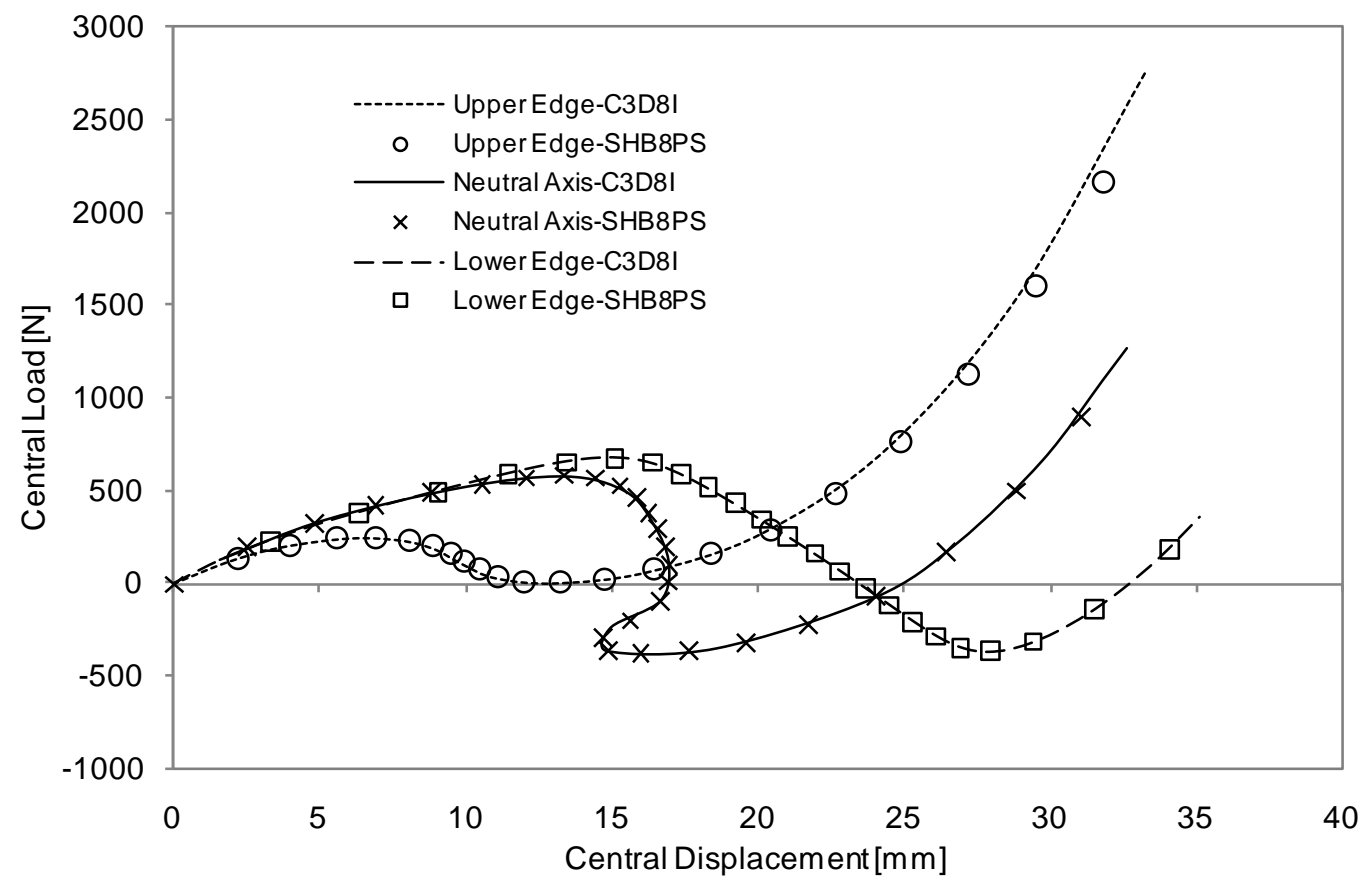


Fig. 22. Load-displacement curves for various boundary conditions for the hinged thin cylindrical section subjected to a concentrated load.

\section{Discussion and conclusions}

In this study a representative set of popular limit-point buckling benchmark problems has been thoroughly investigated. To this end, the recently developed continuum shell finite element SHB8PS has been implemented in the commercial finite element software package Abaqus. This enabled us to use this element for the simulation of several limitpoint buckling benchmark problems and to assess its ability to deal with this type of problem, as compared to relevant elements available in Abaqus. The proposed element successfully passed all the tests. Its performance in terms of accuracy and convergence has proved to be superior to that of linear solid elements and in most cases it was quite comparable to that of state-of-the-art shell elements. Moreover, the three-dimensional geometry of this newly developed solid-shell element allows for an accurate description of the real boundary conditions which was shown to strongly affect the solution in several practical applications.

\section{Acknowledgements}

This work has been carried out as part of a project funded by Agence Nationale de la Recherche, France (contract ANR-005-RNMP-007). The authors are grateful to Professor Alain Combescure for fruitful discussions during the preparation of this work.

\section{References}

[1] E. Domissy, Formulation et évaluation d'éléments finis volumiques modifiés pour l'analyse linéaire et non linéaire des coques. PhD thesis, UT Compiègne, France (1997).

[2] C. Cho, H.C. Park, S.W. Lee, Stability analysis using a geometrically nonlinear assumed strain solid shell element model. Finite Elements Anal Des 29 (1998) 121135.

[3] R. Hauptmann, K. Schweizerhof, A systematic development of solid-shell element formulations for linear and non-linear analyses employing only displacement degrees of freedom. Int J Numer Methods Eng 42 (1998) 49-69.

[4] D. Lemosse, Eléments finis isoparamétriques tridimensionnels pour l'étude des structures minces. PhD thesis, INSA-Rouen, France (2000). 
[5] K.Y. Sze, L.Q., Yao, A hybrid stress ANS solid-shell element and its generalization for smart structure modelling. Part I-solid-shell element formulation. Int J Numer Methods Eng 48 (2000) 545-564.

[6] R. Hauptmann, S. Doll, M. Harnau, K. Schweizerhof, Solid-shell elements with linear and quadratic shape functions at large deformations with nearly incompressible materials. Comput Struct 79 (2001) 1671-1685.

[7] F. Abed-Meraim, A. Combescure, SHB8PS a new intelligent assumed strain continuum mechanics shell element for impact analysis on a rotating body. In: First MIT conference on computational fluid and solid mechanics. Cambridge, USA, 2001.

[8] F. Abed-Meraim, A. Combescure, SHB8PS-a new adaptive, assumed-strain continuum mechanics shell element for impact analysis. Comput Struct 80 (2002) 791-803.

[9] A. Legay, A. Combescure, Elastoplastic stability analysis of shells using the physically stabilized finite element SHB8PS. Int J Numer Methods Eng 57 (2003) 1299-1322.

[10] J.C. Simo, M.S. Rifai, A class of mixed assumed strain methods and the method of incompatible modes. Int J Numer Methods Eng 29 (1990) 1595-1638.

[11] J.C. Simo, F. Armero, Geometrically non-linear enhanced strain mixed methods and the method of incompatible modes. Int J Numer Methods Eng 33 (1992) 1413-1449.

[12] J.C. Simo, F. Armero, R.L. Taylor, Improved versions of assumed enhanced strain trilinear elements for 3D finite deformation problems. Comput Methods Appl Mech Eng 110 (1993) 359-386.

[13] L. Vu-Quoc, X.G. Tan, Optimal solid shells for non-linear analyses of multilayer composites. I. Statics. Comput Methods Appl Mech Eng 192 (2003) 975-1016.

[14] Y.I. Chen, G.Y. Wu, A mixed 8-node hexahedral element based on the Hu-Washizu principle and the field extrapolation technique. Struct Eng Mech 17 (2004) 113-140.

[15] K.D. Kim, G.Z. Liu, S.C. Han, A resultant 8-node solid-shell element for geometrically nonlinear analysis. Comput Mech 35 (2005) 315-331.

[16] R.J. Alves de Sousa, R.P.R. Cardoso, R.A. Fontes Valente, J.W. Yoon, J.J. Gracio, R.M. Natal Jorge, A new one-point quadrature enhanced assumed strain (EAS) solidshell element with multiple integration points along thickness: Part I - Geometrically linear applications. Int J Numer Methods Eng 62 (2005) 952-977.

[17] R.J. Alves de Sousa, R.P.R. Cardoso, R.A. Fontes Valente, J.W. Yoon, J.J. Gracio, R.M. Natal Jorge, A new one-point quadrature enhanced assumed strain (EAS) solidshell element with multiple integration points along thickness: Part II - Nonlinear applications. Int J Numer Methods Eng 67 (2006) 160-188.

[18] S. Reese, A large deformation solid-shell concept based on reduced integration with hourglass stabilization. Int J Numer Methods Eng 69 (2007) 1671-1716.

[19] F. Abed-Meraim, A. Combescure, An improved assumed strain solid-shell element formulation with physical stabilization for geometric nonlinear applications and elastic-plastic stability analysis. Int J Numer Methods Eng 80 (2009) 1640-1686. 
[20] T. Belytschko, L.P. Bindeman, Assumed strain stabilization of the eight node hexahedral element. Comput Methods Appl Mech Eng 105 (1993) 225-260.

[21] J.C. Simo, T.J.R. Hughes, On the variational foundations of assumed strain methods. ASME J Appl Mech 53 (1986) 51-54.

[22] D.P. Flanagan, T. Belytschko, A uniform strain hexahedron and quadrilateral with orthogonal hourglass control. Int J Numer Methods Eng 17 (1981) 679-706.

[23] S.P. Timoshenko, J.M. Gere, Theory of elastic stability. New York: McGraw-Hill (1961).

[24] W.T. Koiter, On the stability of elastic equilibrium. PhD thesis, Delft (1945). English translation NASA Tech Trans F10 (1967).

[25] J.W. Hutchinson, W.T. Koiter, Post-buckling theory. Appl Mech Rev 23 (1970) 13531366.

[26] J.M.T. Thompson, G.W. Hunt, A General theory of elastic Stability. New York: Wiley (1973).

[27] B. Budiansky, Theory of buckling and post-buckling behaviour of elastic structures. Adv Appl Mech 14 (1974) 1-65.

[28] ABAQUS Version 6.7 Documentation, Dassault Systèmes Simulia Corp. (2007).

[29] E. Riks, An incremental approach to the solution of snapping and buckling problems. Int J Solids Struct 15 (1979) 529-551.

[30] M.A. Crisfield, A fast incremental/iterative solution procedure that handles "snapthrough". Comput Struct 13 (1981) 55-62.

[31] E. Ramm, Strategies for tracing the nonlinear response near limit points. In: W. Wunderlich, E. Stein, K.J. Bathe, editors. Nonlinear finite element analysis in structural mechanics. New York: Springer-Verlag (1981) pp. 63-89.

[32] I. Leahu-Aluas, F. Abed-Meraim, A proposed set of popular limit point buckling benchmark problems. Struct Eng Mech (2011) submitted.

[33] P. Wriggers, J.C. Simo, A general procedure for the direct computation of turning and bifurcation points. Int J Numer Methods Eng 30 (1990) 155-176.

[34] L. Jiang, M.W. Chernuka, A corotational formulation for geometrically nonlinear finite element analysis of spatial beams. Trans Canadian Soc Mech Eng 18 (1994) 65-88.

[35] E.H. Boutyour, H. Zahrouni, M. Potier-Ferry, M. Boudi, Bifurcation points and bifurcated branches by an asymptotic numerical method and Padé approximants. Int $J$ Numer Methods Eng 60 (2004) 1987-2012.

[36] K.S. Surana, Geometrically nonlinear formulation for curved shell elements. Int J Numer Methods Eng 19 (1983) 581-615.

[37] J.C. Simo, L. Vu-Quoc, A three-dimensional finite-strain rod model. Part II: Computational aspects. Comput Methods Appl Mech Eng 58 (1986) 79-116.

[38] D.A. DaDeppo, R. Schmidt, Instability of clamped-hinged circular arches subjected to a point load. Trans ASME J Appl Mech 42 (1975) 894-896. 
[39] J.K. Kim, Y.H. Kim, A predictor-corrector method for structural nonlinear analysis. Comput Methods Appl Mech Eng 191 (2001) 959-974.

[40] S. Klinkel, W. Wagner, A geometrical non-linear brick element based on the EASmethod. Int J Numer Methods Eng 40 (1997) 4529-4545.

[41] K.Y. Sze, S.J. Zheng, A stabilized hybrid-stress solid element for geometrically nonlinear homogeneous and laminated shell analyses. Comput Methods Appl Mech Eng 191 (2002) 1945-1966.

[42] K.Y. Sze, X.H. Liu, S.H. Lo, Popular benchmark problems for geometric nonlinear analysis of shells. Finite Elements Anal Des 40 (2004) 1551-1569. 